\title{
Reference-free time-based localization for an asynchronous target
}

\author{
Yiyin Wang ${ }^{*}$ and Geert Leus
}

\begin{abstract}
Low-complexity least-squares (LS) estimators based on time-of-arrival (TOA) or time-difference-of-arrival (TDOA) measurements have been developed to locate a target node with the help of anchors (nodes with known positions). They require to select a reference anchor in order to cancel nuisance parameters or relax stringent synchronization requirements. Thus, their localization performance relies heavily on the reference selection. In this article, we propose several reference-free localization estimators based on TOA measurements for a scenario, where anchor nodes are synchronized, and the clock of the target node runs freely. The reference-free LS estimators that are different from the reference-based ones do not suffer from a poor reference selection. Furthermore, we generalize existing reference-based localization estimators using TOA or TDOA measurements, which are scattered over different research areas, and we shed new light on their relations. We justify that the optimal weighting matrix can compensate the influence of the reference selection for reference-based weighted LS (WLS) estimators using TOA measurements, and make all those estimators identical. However, the optimal weighting matrix cannot decouple the reference dependency for reference-based WLS estimators using a nonredundant set of TDOA measurements, but can make the estimators using the same set identical as well. Moreover, the Cramér-Rao bounds are derived as benchmarks. Simulation results corroborate our analysis.
\end{abstract}

\section{Introduction}

Localization is a challenging research topic under investigation for many decades. It finds applications in the global positioning system (GPS) [1], radar systems [2], underwater systems [3], acoustic systems [4,5], cellular networks [6], wireless local area networks (WLANs) [7], wireless sensor networks (WSNs) [8,9], etc. It is embraced everywhere at any scale. New applications of localization are continuously emerging, which motivates further exploration and attracts many researchers from different research areas, such as geophysics, signal processing, aerospace engineering, and computer science. In general, the localization problem can be solved by two steps [7-9]: firstly measure the metrics bearing location information, the so-called ranging or bearing, and secondly estimate the positions based on those metrics, the so-called location information fusion. There are mainly four metrics: time-of-arrival (TOA) or time-of-flight (TOF) [10], time-difference-of-arrival (TDOA) [4,11], angle-of-arrival (AOA) [12], and received signal strength

\footnotetext{
* Correspondence: yiyin.wang@tudelft.nl

Faculty of Electrical Engineering, Mathematics and Computer Science, Delft University of Technology, Mekelweg 4, 2628CD Delft, The Netherlands
}

(RSS) [13]. The ranging methods using RSS can be implemented by energy detectors, but they can only achieve a coarse resolution. Antenna arrays are required for AOAbased methods, which encumbers their popularity. On the other hand, the high accuracy and potentially low cost implementation make TOA or TDOA based on ultra-wideband impulse radios (UWB-IRs) a promising ranging method [8].

Closed-form localization solutions based on TOAs or TDOAs are used to locate a target node with the help of anchors (nodes with known positions). They are appreciated for real-time localization applications, initiating iterative localization algorithms, and facilitating Kalman tracking [14]. They have much lower complexity compared to the optimal maximum likelihood estimator (MLE), and also do not require prior knowledge of noise statistics. However, a common feature of existing closedform localization solutions is reference dependency. The reference here indicates the time associated with the reference anchor. For instance, in order to measure TDOAs, a reference anchor has to be chosen first [7]. The reference anchor is also needed to cancel nuisance parameters in closed-form solutions based on TOAs or 
TDOAs [15]. Thus, the localization performance depends heavily on the reference selection. There are some efforts to improve the reference selection [16-18], but they mainly rely on heuristics. Furthermore, when TOAs are measured using the one-way ranging protocol for calculating the distance between the target and the anchor, stringent synchronization is required between these two nodes in the conventional methods $[7,10]$. However, it is difficult to maintain synchronization due to the clock inaccuracy and other error sources. Therefore, various closed-form localization methods resort to using TDOA measurements to relax this synchronization constraint between the target and the anchor. These methods only require synchronization among the anchors, e.g., the source localization methods based on TDOAs using a passive sensor array [4,19-22]. ${ }^{\mathrm{a}}$

In this article, we also relax the above synchronization requirement, and consider a scenario, where anchor nodes are synchronized, and the clock of the target node runs freely. However, instead of using TDOAs, we model the asynchronous effect as a common bias, and propose reference-free least-squares (LS), weighted LS (WLS), and constrained WLS (CWLS) localization estimators based on TOA measurements. Furthermore, we generalize existing reference-based localization solutions using TOA or TDOA measurements, which are scattered over different research areas, and provide new insights into their relations, which have been overlooked. We clarify that the reference dependency for reference-based WLS estimators using TOA measurements can be decoupled by the optimal weighting matrix, which also makes all those estimators identical. However, the influence of the reference selection for reference-based WLS estimators using a nonredundant set of TDOA measurements cannot be compensated by the optimal weighting matrix. But the optimal weighting matrix can make the estimators using the same set equivalent as well. Moreover, the Cramér-Rao bounds (CRBs) are derived as benchmarks for comparison.

The rest of this article is organized as follows. In Section 2, different kinds of reference-free TOA-based estimators are proposed, as well as existing reference-based estimators using TOA measurements. Their relations are thoroughly investigated. In Section 3, we generalize existing reference-based localization algorithms using TDOA measurements, and shed light on their relations as well. Simulation results and performance bounds are shown in Section 4. Conclusions are drawn at the end of the article.

Notation: We use upper (lower) bold face letters to denote matrices (column vectors). $[\mathbf{X}]_{m, n},[\mathbf{X}]_{m,:}$ and $[\mathbf{X}]_{:, n}$ denote the element on the $m$ th row and $n$th column, the $m$ th row, and the $n$th column of the matrix $\mathbf{X}$, respectively. $[\mathbf{x}]_{n}$ indicates the $n$th element of $\mathbf{x} . \mathbf{0}_{m}\left(\mathbf{1}_{m}\right)$ is an all-zero (all-one) column vector of length $m . \mathbf{I}_{m}$ indicates an identity matrix of size $m \times m$. Moreover, $(\cdot)^{T},\|\cdot\|$, and $\odot$ designate transposition, $\ell_{2}$ norm, and element-wise product, respectively. All other notation should be self-explanatory.

\section{Localization based on TOA measurements}

Considering $M$ anchor nodes and one target node, we would like to estimate the position of the target node. All the nodes are distributed in an $l$-dimensional space, e.g., $l=2$ (a plane (2-D)) or $l=3$ (a space (3-D)). The coordinates of the anchor nodes are known and defined as $\mathbf{X}_{a}=$ $\left[\mathbf{x}_{1}, \mathbf{x}_{2}, \ldots, \mathbf{x}_{M}\right]$, where the vector $\mathbf{x}_{i}=\left[x_{1, i}, x_{2}, i, \ldots, \mathbf{x}_{l, i}\right]^{T}$ of length $l$ indicates the known coordinates of the $i$ th anchor node. We employ a vector $\mathbf{x}$ of length $l$ to denote the unknown coordinates of the target node. Our method can also be extended for multiple target nodes. We remark that in a large scale WSN, it is common to localize target nodes in a sequential way [23]. The target nodes that have enough anchors are localized first. Then, the located target nodes can be viewed as new anchors that can facilitate the localization of other target nodes. Therefore, the multiple-anchors-one-target scenario here is of practical interest. We can even consider a case with a moving anchor, in which a ranging signal is periodically transmitted by the target node, and all the positions where the moving anchor receives the ranging signal are viewed as the fixed positions of some virtual anchors. We assume that all the anchors are synchronized, and their clock skews are equal to 1 , whereas the clock of the target node runs freely. Furthermore, we assume that the target node transmits a ranging signal, and all the anchors act as receivers. We remark that other systems may share the same data model such as a passive sensor array for source localization or a GPS system, where a GPS receiver locates itself by exploring the received ranging signals from several satellites [1]. All the satellites are synchronized to an atomic clock, but the GPS receiver has a clock offset relative to the satellite clock. Note that this is a stricter synchronization requirement than ours, as we allow the clock of the target node to run freely. Every satellite sends a ranging signal and a corresponding transmission time. The GPS receiver measures the TOAs, and calculates the time-of-flight (TOF) plus an unknown offset. In this section, TOA measurements are used, and TDOA measurements are employed in the next section.

\subsection{System model}

In this section, all localization algorithms are based on TOA measurements. When the target node transmits a ranging signal, all the anchors receive it and record a timestamp upon the arrival of the ranging signal independently. We define a vector $\mathbf{u}$ of length $M$ to collect all the distances corresponding to the timestamps, 
which is given by $\mathbf{u}=\left[u_{1}, u_{2}, \ldots, u_{M}\right]^{T}$. We employ $b$ to denote the distance corresponding to the true target node transmission instant, which is unknown. We remark that if we consider a GPS system, then $\mathbf{u}$ collects the distances corresponding to the biased TOFs calculated by the GPS receiver, and $b$ indicates the distance bias corresponding to the unknown clock offset of the GPS receiver relative to the satellite. Consequently, the TOA measurements can be modeled as

$$
\mathbf{u}-b \mathbf{1}_{M}=\mathbf{d}+\mathbf{n},
$$

where $\mathbf{d}=\left[d_{1}, d_{2}, \ldots, d_{\mathrm{M}}\right]^{\mathrm{T}}$, with $d_{i}=\left\|\mathbf{x}_{i}-\mathbf{x}\right\|$ the true distance between the $i$ th anchor node and the target node, and $\mathbf{n}=\left[n_{1}, n_{2}, \ldots, n_{M}\right]^{T}$ with $n_{i}$ the distance error term corresponding to the TOA measurement error at the $i$ th anchor, which can be modeled as a random variable with zero mean and variance $\sigma_{i}^{2}$, and which is independent of the other terms $\left(E\left[n_{i} n_{j}\right]=0, i \neq j\right)$. We remark that instead of using TDOAs to directly get rid of the distance bias, we use TOAs and take the bias into account in the system model.

\subsection{Localization based on squared TOA measurements 2.2.1. Proposed localization algorithms}

Note that (1) is a nonlinear equation with respect to (w.r.t.) x. To solve it, a MLE can be derived, which is optimal in the sense that for a large number of data it is unbiased and approaches the CRB. However, the MLE has a high computational complexity, and also requires the unknown noise statistics. Therefore, lowcomplexity solutions are of great interest for localization. From $\left\|\mathbf{x}_{i}-\mathbf{x}\right\|^{2}=\left\|\mathbf{x}_{i}\right\|^{2}-2 \mathbf{x}_{i}^{T} \mathbf{x}+\|\mathbf{x}\|^{2}$, we derive that $\quad \mathbf{d} \odot \mathbf{d}=\psi_{a}-2 \mathbf{X}_{a}^{T} \mathbf{x}+\|\mathbf{x}\|^{2} \mathbf{1}_{M}, \quad$ where $\psi_{a}=\left[\left\|\mathbf{x}_{1}\right\|^{2},\left\|\mathbf{x}_{2}\right\|^{2}, \ldots,\left\|\mathbf{x}_{M}\right\|^{2}\right]^{T}$. Element-wise multiplication at both sides of (1) is carried out, which leads to

$$
\mathbf{u} \odot \mathbf{u}-2 b \mathbf{u}+b^{2} \mathbf{1}_{M}=\boldsymbol{\psi}_{\mathbf{a}}-2 \mathbf{X}_{a}^{T} \mathbf{x}+\|\mathbf{x}\|^{2} \mathbf{1}_{M}+2 \mathbf{d} \odot \mathbf{n}+\mathbf{n} \odot \mathbf{n} .
$$

Moving knowns to one side and unknowns to the other side, we achieve

$$
\psi_{a}-\mathbf{u} \odot \mathbf{u}=2 \mathbf{X}_{a}^{T} \mathbf{x}-2 b \mathbf{u}+\left(b^{2}-\|\mathbf{x}\|^{2}\right) \mathbf{1}_{M}+\mathbf{m},
$$

where $\mathbf{m}=-(2 \mathbf{d} \odot \mathbf{n}+\mathbf{n} \odot \mathbf{n})$. The stochastic properties of $\mathbf{m}$ are as follows

$$
\begin{aligned}
& E\left[[\mathbf{m}]_{i}\right]=-\sigma_{i}^{2} \approx 0, \\
& {[\boldsymbol{\Sigma}]_{i, j}=E\left[[\mathbf{m}]_{i}[\mathbf{m}]_{j}\right]-E\left[[\mathbf{m}]_{i}\right] E\left[[\mathbf{m}]_{j}\right]} \\
& =E\left[\left(2 d_{i} n_{i}+n_{i}^{2}\right)\left(2 d_{j} n_{j}+n_{j}^{2}\right)\right]-\sigma_{i}^{2} \sigma_{j}^{2} \\
& =4 d_{i} d_{j} E\left[n_{i}, n_{j}\right]+E\left[n_{i}^{2} n_{j}^{2}\right]-\sigma_{i}^{2} \sigma_{j}^{2} \\
& =\left\{\begin{array}{ll}
4 d_{i}^{2} \sigma_{i}^{2}+2 \sigma_{i}^{4} \approx 4 d_{i}^{2} \sigma_{i}^{2}, & i=j \\
0, & i \neq j
\end{array},\right.
\end{aligned}
$$

where we ignore the higher order noise terms to obtain (5) and assume that the noise mean $E\left[[\mathbf{m}]_{i}\right] \approx 0$ under the condition of sufficiently small measurement errors. Note that the noise covariance matrix $\boldsymbol{\Sigma}$ depends on the unknown $\mathbf{d}$.

Defining $\boldsymbol{\varphi}=\boldsymbol{\psi}_{\mathbf{a}}-\mathbf{u} \odot \mathbf{u}, \mathbf{y}=\left[\mathbf{x}^{T}, b, b^{2}-\|\mathbf{x}\|^{2}\right]^{T}$, and $\mathbf{A}=\left[2 \mathbf{X}_{a}^{T},-2 \mathbf{u}, \mathbf{1}_{M}\right]$, we can finally rewrite (3) as

$$
\phi=\text { Ay }+\mathbf{m} \text {. }
$$

Ignoring the parameter relations in $\mathbf{y}$, an unconstrained LS and WLS estimate of $\mathbf{y}$ can be computed respectively given by

$$
\hat{\mathbf{y}}=\left(\mathbf{A}^{T} \mathbf{A}\right)^{-1} \mathbf{A}^{T} \boldsymbol{\phi}
$$

and

$$
\hat{\mathbf{y}}=\left(\mathbf{A}^{T} \mathbf{W A}\right)^{-1} \mathbf{A}^{T} \mathbf{W} \boldsymbol{\phi},
$$

where $\mathbf{W}$ is a weighting matrix of size $M \times M$. Note that $M \geq l+2$ is required in (7) and (8), which indicates that we need at least four anchors to estimate the target position on a plane. The optimal $\mathbf{W}$ is $\mathbf{W}^{*}=\boldsymbol{\Sigma}^{-1}$, which depends on the unknown $\mathbf{d}$ as we mentioned before. Thus, we can update it iteratively, and the resulting iterative WLS can be summarized as follows:

(1) Initialize $\mathbf{W}$ using the estimate of $\mathbf{d}$ based on the LS estimate of $\mathbf{x}$;

(2) Estimate $\hat{\mathbf{y}}$ using (8);

(3) Update $\mathbf{W}=\hat{\mathbf{\Sigma}}^{-1}$ where $\hat{\boldsymbol{\Sigma}}$ is computed using $\hat{\mathbf{y}}$;

(4) Repeat Steps (2) and (3) until a stopping criterion is satisfied.

The typical stopping criteria are discussed in [24]. We stop the iterations when $\left\|\hat{\mathbf{y}}^{(k+1)}-\hat{\mathbf{y}}^{(k)}\right\| \leq \varepsilon$, where $\hat{\mathbf{y}}^{(k)}$ is the estimate of the $k$ th iteration and $\epsilon$ is a given threshold [25]. An estimate of $\mathbf{x}$ is finally given by

$$
\hat{\mathbf{x}}=\left[\begin{array}{ll}
\mathbf{I}_{l} & \mathbf{0}_{l \times 2}
\end{array}\right] \hat{\mathbf{y}}
$$

To accurately estimate $\mathbf{y}$, we can further explore the relations among the parameters in y. A CWLS estimator is obtained as

$$
\hat{\mathbf{y}}=\arg \min _{\hat{\mathbf{y}}}(\phi-\mathbf{A y})^{T} \mathbf{W}(\boldsymbol{\phi}-\mathbf{A y})
$$

subject to

$$
\mathbf{y}^{T} \mathbf{J y}+\boldsymbol{\rho}^{T} \mathbf{y}=0,
$$

where $\rho=\left[\mathbf{0}_{l+1}^{T}, 1\right]^{T}$ and

$$
\mathbf{J}=\left[\begin{array}{lll}
\mathbf{I}_{l} & \mathbf{0}_{l} & \mathbf{0}_{l} \\
\mathbf{0}_{l}^{T} & -1 & 0 \\
\mathbf{0}_{l}^{T} & 0 & 0
\end{array}\right] .
$$


Solving the CWLS problem is equivalent to minimizing the Lagrangian $[4,10]$

$$
\mathcal{L}(\mathbf{y}, \lambda)=(\boldsymbol{\phi}-\mathbf{A y})^{T} \mathbf{W}(\boldsymbol{\phi}-\mathbf{A y})+\lambda\left(\mathbf{y}^{T} \mathbf{J y}+\boldsymbol{\rho}^{T} \mathbf{y}\right),
$$

where $\lambda$ is a Lagrangian multiplier. A minimum point for (13) is given by

$$
\hat{\mathbf{y}}=\left(\mathrm{A}^{T} \mathbf{W A}+\lambda \mathbf{J}\right)^{-1}\left(\mathrm{~A}^{T} \mathbf{W} \boldsymbol{\phi}-\frac{\lambda}{2} \rho\right),
$$

where $\lambda$ is determined by plugging (14) into the following equation

$$
\hat{\mathbf{y}}^{T} \mathbf{J} \hat{\mathbf{y}}+\rho^{T} \hat{\mathbf{y}}=0 .
$$

We could find all the seven roots of $(15)$ as in $[4,10]$, or employ a bisection algorithm as in [26] to look for $\lambda$ instead of finding all the roots. If we obtain seven roots as in $[4,10]$, we discard the complex roots, and plug the real roots into (14). Finally, we choose the estimate $\hat{\mathbf{y}}$, which fulfills (10). The details of solving (15) are mentioned in Appendix 1. Note that the proposed CWLS estimator (14) is different from the estimators in $[4,10]$. The CLS estimator in [4] is based on TDOA measurements, and the CWLS estimator in [10] is based on TOA measurements for a synchronous target $(b=0)$. Furthermore, we remark that the WLS estimator proposed in [27] based on the same data model as (1), is labeled as an extension of Bancroft's algorithm [28], which is actually similar to the spherical-intersection (SX) method proposed in [29] for TDOA measurements. It first solves a quadratic equation in $b^{2}-\|\mathbf{x}\|^{2}$, and then estimates $\mathbf{x}$ and $b$ via a WLS estimator. However, it fails to provide a solution for the quadratic equation under certain circumstances, and performs unsatisfactorily when the target node is far away from the anchors [29].

Many research works have focused on LS solutions ignoring the constraint (11) in order to obtain low-complexity closed-form estimates [7]. As squared range (SR) measurements are employed, we call them unconstrained SR-based LS (USR-LS) approaches, to be consistent with [26]. Because only $\mathbf{x}$ is of interest, $b$ and $b^{2}$ - $\|\mathbf{x}\|^{2}$ are nuisance parameters. Different methods have been proposed to get rid of them instead of estimating them. A common characteristic of all these methods is that they have to choose a reference anchor first, and thus we label them reference-based USR-LS (REFBUSR-LS) approaches. As a result, the performance of these REFB-USR-LS methods depends on the reference selection [7]. However, note that the unconstrained LS estimate of $\mathbf{y}$ in (7) does not depend on the reference selection. Thus, we call (7) the reference-free USR-LS (REFF-USR-LS) estimate, (8) the REFF-USR-WLS, and (14) the REFF-SR-CWLS estimate.
Moreover, we propose the subspace minimization (SM) method [22] to achieve a REFF-USR-LS estimate of $\mathbf{x}$ alone, which is identical to $\hat{\mathbf{x}}$ in (7), but shows more insight into the links among different estimators. Treating $b$ and $b^{2}-\|\mathbf{x}\|^{2}$ as nuisance parameters, we try to get rid of them by orthogonal projections instead of random reference selection. We first use an orthogonal projection $\mathbf{P}=\mathbf{I}_{M}-\frac{1}{M} \mathbf{1}_{M} \mathbf{1}_{M}^{T}$ of size $M \times M$ onto the orthogonal complement of $\mathbf{1}_{M}$ to eliminate $\left(b^{2}-\|\right.$ $\left.\mathbf{x} \|^{2}\right) \mathbf{1}_{M}$. Sequentially, we employ a second orthogonal projection $\mathbf{P}_{u}$ of size $M \times M$ onto the orthogonal complement of $\mathbf{P u}$ to cancel $-2 b \mathbf{P u}$, which is given by

$$
\mathbf{P}_{u}=\mathbf{I}_{M}-\frac{\mathbf{P u u}^{T} \mathbf{P}}{\mathbf{u}^{T} \mathbf{P u}} .
$$

Thus, premultiplying (3) with $\mathbf{P}_{u} \mathbf{P}$, we obtain

$$
\mathbf{P}_{u} \mathbf{P} \boldsymbol{\phi}=2 \mathbf{P}_{u} \mathbf{P X} \mathbf{X}_{a}^{T} \mathbf{x} \mathbf{P}_{u} \mathbf{P m},
$$

which is linear w.r.t. x. The price paid for applying these two projections is the loss of information. The rank of $\mathbf{P}_{u} \mathbf{P}$ is $M-2$, which means that $M \geq l+2$ still has to be fulfilled as before to obtain an unconstrained LS or WLS estimate of $\mathbf{x}$ based on (17). In a different way, $\mathbf{P}_{u} \mathbf{P}$ can be achieved directly by calculating an orthogonal projection onto the orthogonal complement of $\left[\mathbf{1}_{M}, \mathbf{u}\right]$. Let us define the nullspace $\mathcal{N}\left(\mathbf{U}^{T}\right)=\operatorname{span}\left(\mathbf{1}_{M}, \mathbf{u}\right)$, and $\mathcal{R}(\mathbf{U}) \oplus \mathcal{N}\left(\mathbf{U}^{T}\right)=\mathbb{R}^{M}$, where $\mathcal{R}(\mathbf{U})$ is the column space of $\mathbf{U}, \oplus$ denotes the direct sum of two linearly independent subspaces and $\mathbb{R}^{M}$ is the $M$-dimensional vector space. Therefore, $\mathbf{P}_{u} \mathbf{P}$ is the projection onto $\mathcal{R}(\mathbf{U})$. Note that the rank of $\mathbf{P}_{u} \mathbf{P X}_{a}^{T}$ has to be equal to $l$, which indicates that the anchors should not be co-linear for both 2$\mathrm{D}$ and 3-D or co-planar for 3-D. A special case occurs when $\mathbf{u}=k \mathbf{1}_{M}$, where $k$ is any positive real number. In this case, $\mathbf{P}$ can cancel out both $\left(b^{2}-\|\mathbf{x}\|^{2}\right) \mathbf{1}_{M}$ and $-2 b \mathbf{u}$, and one projection is enough, leading to the condition $M$ $\geq l+1$. The drawback though is that we can then only estimate $\mathbf{x}$ and $b^{2}-\|\mathbf{x}\|^{2}-2 b k$ due to the dependence between $\mathbf{u}$ and $\mathbf{1}_{M}$ according to (3). The SM method indicates all the insights mentioned above, which cannot be easily observed by the unconstrained estimators.

Based on (17), the LS and WLS estimate of $\mathbf{x}$ is respectively given by,

$$
\hat{\mathbf{x}}=\frac{1}{2}\left(\mathbf{X}_{a} \mathbf{P} \mathbf{P}_{u} \mathbf{P X} \mathbf{X}_{a}^{T}\right)^{-1} \mathbf{X}_{a} \mathbf{P} \mathbf{P}_{u} \mathbf{P} \boldsymbol{\phi}
$$

and

$$
\hat{\mathbf{X}}=\frac{1}{2}\left(\mathbf{X}_{a} \mathbf{Q} \mathbf{X}_{a}^{T}\right)^{-1} \mathbf{X}_{a} \mathbf{Q} \boldsymbol{\phi},
$$


where $\mathbf{Q}$ is an aggregate weighting matrix of size $M \times$ $M$. The optimal $\mathbf{Q}$ is given by

$$
\begin{aligned}
& \mathbf{Q}^{*}=\mathbf{P P}_{u}\left(\mathbf{P}_{u} \mathbf{P} \Sigma \mathbf{P} \mathbf{P}_{u}\right)^{\dagger} \mathbf{P}_{u} \mathbf{P} \\
& =\left(\mathbf{P}_{u} \mathbf{P} \Sigma \mathbf{P} \mathbf{P}_{u}\right)^{\dagger},
\end{aligned}
$$

where the pseudo-inverse $(\dagger)$ is employed, because the argument is rank deficient. Note that $\mathbf{P}_{u} \mathbf{P}$ is the projection onto $\mathcal{R}(\mathbf{U})$, and is applied to both sides of $\boldsymbol{\Sigma}$. Thus, $\left(\mathbf{P}_{u} \mathbf{P} \boldsymbol{\Sigma} \mathbf{P} \mathbf{P}_{u}\right)^{\dagger}$ is still in $\mathcal{R}(\mathbf{U})$, and would not change with applying the projection again. As a result, we can simplify (20) as (21). Consequently, $\mathbf{Q}^{*}$ is the pseudo-inverse of the matrix obtained by projecting the columns and rows of $\boldsymbol{\Sigma}$ onto $\mathcal{R}(\mathbf{U})$, which is of rank $M$ - 2. We remark that $\hat{\mathbf{x}}$ in (18) (or (19)) is identical to the one in (7) (or (8)) according to [22]. The SM method and the unconstrained LS (or WLS) method lead to the same result. Therefore, $\hat{\mathbf{x}}$ in (18) and (7) (or in (19) and (8)) are all REFF-USR-LS (or REFF-USRWLS) estimates.

\subsubsection{Revisiting existing localization algorithms}

As we mentioned before, all the REFB-USR-LS methods suffer from a poor reference selection. There are some efforts to improve the reference selection [16-18]. In [16], the operation employed to cancel $\|\mathbf{x}\|^{2} \mathbf{1}_{M}$ is equivalent to the orthogonal projection $\mathbf{P}$. All anchors are chosen as a reference once in [17] in order to obtain $M(M-1) / 2$ equations in total. A reference anchor is chosen based on the criterion of the shortest anchor-target distance measurement in [18]. However, reference-free methods are better than these heuristic reference-based methods in the sense that they cancel nuisance parameters in a systematic way. To clarify the relations between the REFBUSR and the REFF-USR approaches, we generalize the reference selection of all the reference-based methods as a linear transformation, which is used to cancel nuisance parameters, similarly as an orthogonal projection. To eliminate $\left(b^{2}-\|\mathbf{x}\|^{2}\right) \mathbf{1}_{M}$, the $i$ th anchor is chosen as a reference to make differences. As a result, the corresponding linear transformation $\mathbf{T}_{i}$ of size $(M-1) \times M$ can be obtained by inserting the column vector $-\mathbf{1}_{M-1}$ after the (i-1)th column of $\mathbf{I}_{M-1}$, which fulfills $\mathbf{T}_{i} \mathbf{1}_{M}=\mathbf{0}_{M-}$ ${ }_{1}, i \in\{1, \ldots, M\}$. For example, if the first anchor is chosen as a reference, then $\mathbf{T}_{1}=\left[-\mathbf{1}_{M-1}, \mathbf{I}_{M-1}\right]$. Furthermore, we can write $\mathbf{T}_{i} \mathbf{d}=\mathbf{T}_{i 1} \mathbf{d}-d_{i} \mathbf{1}_{m-1}$, where $\mathbf{T}_{i 1}$ is achieved by replacing the $i$ th column of $\mathbf{T}_{i}$ with the column vector $\mathbf{0}_{M-1}$. Applying $\mathbf{T}_{i}$ to both sides of (3), we arrive at

$$
\mathbf{T}_{i} \boldsymbol{\phi}=2 \mathbf{T}_{i} \mathbf{X}_{a}^{T} \mathbf{x}-2 b \mathbf{T}_{i} \mathbf{u}+\mathbf{T}_{i} \mathbf{m} .
$$

Sequentially, we investigate the second linear transformation $\mathbf{M}_{j}$ of size $(M-2) \times(M-1)$, which fulfills $\mathbf{M}_{j} \mathbf{T}_{i} \mathbf{u}=\mathbf{0}_{M-2}, j \in\{1, \ldots, M\}$ and $j \neq i$. As a result, the nullspace $\quad \mathcal{N}\left(\mathbf{M}_{j} \mathbf{T}_{i}\right)=\operatorname{span}\left(\mathbf{1}_{M}, \mathbf{u}\right)=\mathcal{N}\left(\mathbf{U}^{T}\right), \quad$ and $\mathcal{R}\left(\mathbf{T}_{i}^{T} \mathbf{M}_{j}^{T}\right)=\mathcal{R}(\mathbf{U})$. Note that $b=0$ in $[7,16-18,22,26]$, which means that there is no need to apply $\mathbf{M}_{j}$ in these works. But the double differencing method in [15] is equivalent to employing $\mathbf{M}_{j}$, and thus the results of [15] can be used to design $\mathbf{M}_{j}$. Let us first define a matrix $\overline{\mathbf{T}}_{j 1}$ of size $(M-2) \times(M-1)$ similarly as $\mathbf{T}_{i 1}$ using the column vector $\mathbf{0}_{M-2}$ instead of $\mathbf{0}_{M-1}$. When the $j$ th anchor is chosen as a reference and $j<i$, $\mathbf{M}_{j}$ can be obtained by inserting the column vector - $(1 /$ $\left.\left(u_{j}-u_{i}\right)\right) \mathbf{1}_{M-2}$ after the $(j-1)$ th column of the matrix $\operatorname{diag}\left(\overline{\mathbf{T}}_{j 1}\left(\mathbf{1}_{M-1} \oslash\left(\mathbf{T}_{i} \mathbf{u}\right)\right)\right)$, where $\varnothing$ is element-wise division. If $j>i$, then $\mathbf{M}_{j}$ can be obtained by inserting the column vector $-\left(1 /\left(u_{j}-u_{i}\right)\right) \mathbf{1}_{M-2}$ after the $(j-2)$ th column of the matrix $\operatorname{diag}\left(\overline{\mathbf{T}}_{(j-1) 1}\left(\mathbf{1}_{M-1} \oslash\left(\mathbf{T}_{i} \mathbf{u}\right)\right)\right)$. For example, if the first anchor is chosen to cancel out $\left(b^{2}\right.$ - $\left.\|\mathbf{x}\|^{2}\right) \mathbf{1}_{M}\left(\mathbf{T}_{1}\right.$ is used), and the second anchor is chosen to eliminate $\mathbf{T}_{1} \mathbf{u}$, then $\mathbf{M}_{2}$ is given by

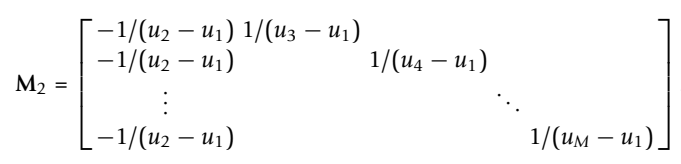

Premultiplying $\mathbf{M}_{j} \mathbf{T}_{i}$ to both sides of (3), we achieve

$$
\mathbf{M}_{j} \mathbf{T}_{i} \phi=2 \mathbf{M}_{j} \mathbf{T}_{i} \mathbf{X}_{a}^{T} \mathbf{x}+\mathbf{M}_{j} \mathbf{T}_{i} \mathbf{m} .
$$

Consequently, the general form of the REFB-USR-LS and the REFB-USR-WLS estimates are derived in the same way as (18) and (19) by replacing $\mathbf{P P}_{u} \mathbf{P}$ and $\mathbf{Q}$ with $\mathbf{T}_{i}^{T} \mathbf{M}_{j}^{T} \mathbf{M}_{j} \mathbf{T}_{i}$ and $\mathbf{Q}_{i, j}$, respectively. We do not repeat these equations for the sake of brevity. Note that $Q_{i, j}$ is an aggregate weighting matrix of size $M \times M$. The optimal $\mathbf{Q}_{i, j}$ is given by

$$
\begin{aligned}
& \mathbf{Q}_{i, j}^{*}=\mathbf{T}_{i}^{T} \mathbf{M}_{j}^{T}\left(\mathbf{M}_{j} \mathbf{T}_{i} \boldsymbol{\Sigma} \mathbf{T}_{i}^{T} \mathbf{M}_{j}^{T}\right)^{-1} \mathbf{M}_{j} \mathbf{T}_{i} \\
& =\left[\left(\mathbf{M}_{j} \mathbf{T}_{i}\right)^{\dagger} \mathbf{M}_{j} \mathbf{T}_{i} \boldsymbol{\Sigma} \mathbf{T}_{i}^{T} \mathbf{M}_{j}^{T}\left(\mathbf{T}_{i}^{T} \mathbf{M}_{j}^{T}\right)^{\dagger}\right]^{\dagger},
\end{aligned}
$$

where $\quad\left(\mathbf{M}_{j} \mathbf{T}_{i}\right)^{\dagger} \mathbf{M}_{j} \mathbf{T}_{i}=\mathbf{T}_{i}^{T} \mathbf{M}_{j}^{T}\left(\mathbf{T}_{i}^{T} \mathbf{M}_{j}^{T}\right)^{\dagger}=\mathbf{T}_{i}^{T} \mathbf{M}_{j}^{T}\left(\mathbf{M}_{j} \mathbf{T}_{i} \mathbf{T}_{i}^{T} \mathbf{M}_{j}^{T}\right)^{-1} \mathbf{M}_{j} \mathbf{T}_{i}$, which is also the projection onto $\mathcal{R}(\mathrm{U})$, and thus is equivalent to $\mathbf{P}_{u} \mathbf{P}$. The equality between (25) and (26) can be verified using a property of the pseudo-inverse. ${ }^{\mathrm{b}}$ Hence, $\mathbf{Q}_{i, j}^{*}$ is of rank $M-2$, and $\mathbf{Q}_{i, j}^{*}=\mathbf{Q}^{*}, i, j \in\{1, \ldots, M\}$ with $i \neq j$. As a result, the REFB-USR-WLS estimate and the REFF-USR-WLS estimate are identical if the optimal weighting matrix is used. Hence, the optimal weighting matrix can compensate the impact of random reference selection. However, since $\boldsymbol{\Sigma}$ depends on the unknown $\mathbf{d}$, the optimal weighting matrix can only be approximated iteratively. Also 
note that the REFB-USR-LS estimate suffers from the ad-hoc reference selection, while the REFF-USR-LS estimate is independent of the reference selection.

\subsection{Localization based on squared differences of TOA measurements}

\subsubsection{Proposed localization algorithms}

Let us recall (1) here, i.e.,

$$
\mathbf{u}-b \mathbf{1}_{M}=\mathbf{d}+\mathbf{n} .
$$

In general, $b$ is regarded as a nuisance parameter. Instead of first carrying out element-wise multiplication at both sides of (27), we can also try to get rid of $b$ before element-wise multiplication. By choosing a reference anchor, and then subtracting the TOAs of other anchors from the TOA of the reference anchor [7], $M$ 1 TDOAs are obtained and $b$ is canceled out. Note that these TDOAs are achieved differently from the TDOAs obtained directly by cross-correlating the received signals from different anchors. The obvious drawback of this conventional scheme is again the reference dependency. On the other hand, since $b$ is a common term in (1), we can again apply $\mathbf{P}$ to eliminate $-b \mathbf{1}_{M}$ instead of randomly choosing a reference anchor. Then we arrive at

$$
\mathbf{P u}=\mathbf{P d}+\mathbf{P n} .
$$

Note that $\mathbf{P u}=\mathbf{u}-\bar{u} \mathbf{1}_{M}$, where $\bar{u}$ is the average TOA. Thus, $\mathbf{P u}$ represents the differences between the anchor TOAs and the average TOA. Moreover, $\mathbf{P d}=\mathbf{d}-\bar{d} \mathbf{1}_{M}$, where $\bar{d}=\frac{1}{M} \sum_{i=1}^{M} d_{i}$ is the unknown average of the distances between the target node and the anchors, and Pn $=\mathbf{n}-\bar{n} \mathbf{1}_{M}$, where $\bar{n}=\frac{1}{M} \sum_{i=1}^{M} n_{i}$. Thus, (28) can be rewritten as

$$
\mathbf{P u}+(\bar{d}+\bar{n}) \mathbf{1}_{M}=\mathbf{d}+\mathbf{n},
$$

By making element-wise multiplication of (29) and rearranging all the terms, we achieve

$$
\psi_{a}-(\mathbf{P u}) \odot(\mathbf{P u})=2 \mathbf{X}_{a}^{T} \mathbf{x}+2 \bar{d} \mathbf{P u}+\left(\bar{d}^{2}-\|\mathbf{x} \mid\|^{2}\right) \mathbf{1}_{M}+\mathbf{m}+\bar{n}^{2} \mathbf{1}_{M}+2 \bar{n}\left(\bar{d} \mathbf{1}_{M}+\mathbf{P u}\right),
$$

where $\psi_{\boldsymbol{a}}=\left[|| \mathbf{x}_{1}\left\|^{2},\right\| \mathbf{x}_{2}\left\|^{2}, \ldots,\right\| \mathbf{x}_{M} \|^{2}\right]^{T}$ and $\mathbf{m}=-(2 \mathbf{d}$ $\odot \mathbf{n}+\mathbf{n} \odot \mathbf{n})$ as before. Using the SM method to obtain an unconstrained LS estimate of $\mathbf{x}$ alone, we employ again two projections $\mathbf{P}$ and $\mathbf{P}_{u}$, and arrive at

$$
\mathbf{P}_{u} \mathbf{P}\left(\psi_{a}-(\mathbf{P u}) \odot(\mathbf{P u})\right)=2 \mathbf{P}_{u} \mathbf{P X} \mathbf{X}_{a}^{T} \mathbf{x}+\mathbf{P}_{u} \mathbf{P m},
$$

the right hand side of which is exactly the same as the one in (17), and thus we can state $\mathbf{P}_{u} \mathbf{P}\left(\boldsymbol{\psi}_{\boldsymbol{a}}-(\mathbf{P u}) \odot\right.$ $(\mathbf{P u}))=\mathbf{P}_{u} \mathbf{P} \boldsymbol{\varphi}$. Note that although (30) is different from
(3), we find that (31) and (17) become equivalent after premultiplying $\mathbf{P}_{u} \mathbf{P}$. Furthermore, $(\mathbf{P u}) \odot(\mathbf{P u})$ can be labeled as a SR difference (SRD) term. As a result, the unconstrained LS and WLS estimate of $\mathbf{x}$ based on (31), which are named the reference-free USRD-LS (REFFUSRD-LS) estimate and the REFF-USRD-WLS estimate, are exactly the same as the REFF-USR-LS estimate (18) and the REFF-USR-WLS estimate (19), respectively. We do not repeat them here in the interest of brevity. Moreover, the constrained LS and WLS based on (30), namely the REFF-SRD-CLS estimate and the REFFSRD-CWLS estimate, are identical to the REFF-SR-CLS and the REFF-SR-CWLS estimate (14) as well.

\subsubsection{Revisiting existing localization algorithms}

Existing methods choose a reference anchor to obtain range differences, and further investigate low-complexity closed-form LS or WLS solutions. Thus, we call them reference-based USRD-LS (REFB-USRD-LS) and REFBUSRD-WLS approaches. To expose interesting links among the different reference-based or reference-free SR-based or SRD-based approaches, we generalize the conventional REFB-USRD-LS and REFB-USRD-WLS approaches [7] in the same way as in Section 2.2.2. The reference selection can be generalized by a linear transformation similarly as in Section 2.2.2. In order to eliminate $-b \mathbf{1}_{M}$ in (27), the $i$ th anchor is chosen as a reference, thus $\mathbf{T}_{i}$ defined in Section 2.2.2 is employed, which fulfills $\mathbf{T}_{i} \mathbf{1}_{M}=\mathbf{0}_{M-1}$. Applying $\mathbf{T}_{i}$ instead of $\mathbf{P}$ to (27), following the same operations to obtain (30), and noting that $\left(\mathbf{T}_{i 1}(\mathbf{d}+\mathbf{n})\right) \odot\left(\mathbf{T}_{i 1}(\mathbf{d}+\mathbf{n})\right)=\mathbf{T}_{i 1}((\mathbf{d}+\mathbf{n})$ $\odot(\mathbf{d}+\mathbf{n}))$, we arrive at

$$
\mathbf{T}_{i} \psi_{a}-\left(\mathbf{T}_{i} \mathbf{u}\right) \odot\left(\mathbf{T}_{i} \mathbf{u}\right)=2 \mathbf{T}_{i} \mathbf{X}_{a}^{T} \mathbf{x}+2 d_{i} \mathbf{T}_{i} \mathbf{u}+\mathbf{T}_{i} \mathbf{m}+2 n_{i} \mathbf{T}_{i} \mathbf{u},
$$

which is different from (30), and has only one nuisance parameter $d_{i}$ at the right hand side. Ignoring the relation between $\mathbf{x}$ and $d_{i}$, we still have two ways to deal with $d_{i}$. The first one is to estimate $\mathbf{x}$ and $d_{i}$ together [22], which means we only use a reference once for calculating the TDOAs. The second one is again to apply $\mathbf{M}_{j}$, which fulfills $\mathbf{M}_{j} \mathbf{T}_{i} \mathbf{u}=\mathbf{0}_{M-2}$. It employs two different references, one for calculating the TDOAs, and the other for eliminating the nuisance parameter. In order to distinguish these two, we call them the REFB-USRDLS(1) and the REFB-USRD-LS(2) estimate, respectively, where the number between brackets indicates the number of references used in the approach. In the same way as we clarified the equivalence between the REFFUSRD-LS and the REFF-USR-LS estimate in the previous subsection, we can easily confirm the equivalence between the REFB-USRD-LS(2) (or the REFB-USRDWLS(2)) and the REFB-USR-LS (or the REFB-USRWLS) estimate of Section 2.2.2. We omit the details for the sake of brevity. Furthermore, we recall that similarly 
as above we could have dealt with $-2 b \mathbf{T}_{i} \mathbf{u}$ in (22) in two different ways. But since $b=0$ in $[7,16-18,22,26]$, there are no discussions about these two different ways in literature, and we do not distinguish between them in the REFB-USR-LS method.

Since there is no counterpart of the REFB-USRD-LS(1) estimate in Section 2.2.2 for the SR-based methods, we briefly discuss the REFB-USRD-LS(1) estimate to complete the investigation of the links among all the estimators based on TOA measurements. Employing the SM method, we again use an orthogonal projection $\mathbf{P}_{i}$ of size $(M-1) \times$ $(M-1)$ onto the orthogonal complement of $\mathbf{T}_{i} \mathbf{u}$ to fulfill $\mathbf{P}_{i} \mathbf{T}_{i} \mathbf{u}=0_{M-1}$, which can be derived in the same way as (16) by replacing $\mathbf{I}_{M}$ and $\mathbf{P u}$ with $\mathbf{I}_{M-1}$ and $\mathbf{T}_{i} \mathbf{u}$, respectively. As a result, $\mathcal{N}\left(\mathbf{P}_{i} \mathbf{T}_{i}\right)=\operatorname{span}\left(\mathbf{1}_{M}, \mathbf{u}\right)=\mathcal{N}\left(\mathbf{U}^{T}\right)$ and $\mathcal{R}\left(\mathbf{T}_{i}^{T} \mathbf{P}_{i}\right)=\mathcal{R}(\mathbf{U})$. Premultiplying (32) with $\mathbf{P}_{i}$, we obtain

$$
\mathbf{P}_{i} \mathbf{T}_{i} \boldsymbol{\psi}_{\mathbf{a}}-\mathbf{P}_{i}\left(\left(\mathbf{T}_{i} \mathbf{u}\right) \odot\left(\mathbf{T}_{i} \mathbf{u}\right)\right)=2 \mathbf{P}_{i} \mathbf{T}_{i} \mathbf{X}_{a}^{T} \mathbf{x}+\mathbf{P}_{i} \mathbf{T}_{i} \mathbf{m} .
$$

Note that $\mathbf{P}_{i}\left(\left(\mathbf{T}_{i} \mathbf{u}\right) \odot\left(\mathbf{T}_{i} \mathbf{u}\right)\right)=\mathbf{P}_{i} \mathbf{T}_{i}(\mathbf{u} \odot \mathbf{u})$ (see Appendix 2 for a proof), and thus we can state $\mathbf{P}_{i} \mathbf{T}_{i \psi a}$ $\left.-\mathbf{P}_{\mathrm{i}}\left(\left(\mathbf{T}_{\mathrm{i}} \mathbf{u}\right) \odot\left(\mathbf{T}_{i} \mathbf{u}\right)\right)=\mathbf{P}_{i} \mathbf{T}_{i \boldsymbol{\varphi}}\right)$. Consequently, the REFBUSRD-LS(1) and the REFB-USRD-WLS(1) estimates can also be written as (18) and (19) by replacing $\mathbf{P P}_{u} \mathbf{P}$ and $\mathbf{Q}$ with $\mathbf{T}_{i}^{T} \mathbf{P}_{i} \mathbf{T}_{i}$ and $\mathbf{Q}_{i}$, respectively. We do not repeat the equations in the interest of brevity. We remark that $\mathbf{Q}_{i}$ is again an aggregate weighting matrix of size $M \times M$, and the optimal $\mathbf{Q}_{i}$ of rank $(M-2)$ is given by

$$
\begin{aligned}
& \mathbf{Q}_{i}^{*}=\mathbf{T}_{i}^{T} \mathbf{P}_{i}\left(\mathbf{P}_{i} \mathbf{T}_{i} \boldsymbol{\Sigma} \mathbf{T}_{i}^{T} \mathbf{P}_{i}\right)^{\dagger} \mathbf{P}_{i} \mathbf{T}_{i} \\
& =\left(\mathbf{V}_{i} \mathbf{V}_{i}^{T} \boldsymbol{\Sigma} \mathbf{V}_{i} \mathbf{V}_{i}^{T}\right)^{\dagger},
\end{aligned}
$$

where $\mathbf{V}_{i}$ is of size $M \times(M-2)$, and collects the right singular vectors corresponding to the $M-2$ nonzero singular values of $\mathbf{P}_{i} \mathbf{T}_{i}$. We derive (35) in Appendix 3, and prove that $\mathbf{V}_{i} \mathbf{V}_{i}^{T}$ is the projection onto $\mathcal{R}(\mathbf{U})$. As a result, $\mathbf{Q}_{i}^{*}=\mathbf{Q}_{i, j}^{*}=\mathbf{Q}^{*}, i, j \in\{1, \ldots, M\}$ and $i \neq j$.

Based on the above discussions, we achieve the important conclusion that the REFF-USRD-WLS, the REFBUSRD-WLS(1), the REFB-USRD-WLS(2), the REFFUSR-WLS, and the REFB-USR-WLS estimate are all identical if the optimal weighting matrix is adopted. The optimal weighting matrix releases the reference-based methods from the influence of a random reference selection. Moreover, the REFF-USR-LS and the REFFUSRD-LS estimate are identical, and free from a reference selection, whereas the REFB-USR-LS and the REFB-USRD-LS(2) estimate are equivalent, but still suffer from a poor reference selection.

To further improve the localization accuracy, a constrained WLS estimate based on (32) can be pursued considering the relation between $\mathbf{x}$ and $d_{i}$ similarly as in
[26]. We call it the reference-based SRD CWLS (REFBSRD-CWLS) estimate. Denoting $\mathbf{z}=\left[\mathbf{x}^{T}, d_{i}\right]^{T}, \mathbf{B}_{i}=2 \mathbf{T}_{i}\left[\mathbf{X}_{a}^{T}, \mathbf{u}\right]$ and $\varrho_{i}=\mathbf{T}_{i} \psi_{\boldsymbol{a}}-\left(\mathbf{T}_{i} \mathbf{u}\right) \odot$ $\left(\mathbf{T}_{i} \mathbf{u}\right)$, it is given by,

$$
\hat{\mathbf{z}}=\arg \min _{\hat{\mathbf{z}}}\left(\rho_{i}-\mathbf{B}_{i} \mathbf{z}\right)^{T} \mathbf{W}_{i}\left(\rho_{i}-\mathbf{B}_{i} \mathbf{z}\right)
$$

subject to

$$
\left(\mathbf{z}-\mathbf{z}_{i}\right)^{T} \mathbf{L}\left(\mathbf{z}-\mathbf{z}_{i}\right)=0 \quad \text { and } \quad[\mathbf{z}]_{l+1} \geq 0,
$$

where $\mathbf{W}_{i}$ is a weighting matrix of size $(M-1) \times(M-$ 1), $\mathbf{z}_{i}=\left[\begin{array}{ll}\mathbf{x}_{i}^{T} & 0\end{array}\right]^{T}$ and

$$
\mathbf{L}=\left[\begin{array}{cc}
\mathbf{I}_{l} & \mathbf{0}_{l} \\
\mathbf{0}_{l}^{T} & -1
\end{array}\right] \text {. }
$$

The method to solve this CWLS problem is proposed in [26]. We do not review it for the sake of brevity. Note that there are two constraints for (36) compared to one for (10), thus the method to solve (36) is different from the one to solve (10).

All the estimators based on TOA measurements are summarized in Tables 1, 2, and 3. They are characterized by the number of references, the reference dependency, the minimum number of anchors, and the optimal weighting matrices. We also shed light on their relations and categorize the existing methods from literature. We remark that the authors in [30] claim that the error covariance of the optimal position estimate using TOAs with a distance bias is equivalent to the one using TDOAs regardless of the reference selection, where the error covariance is defined as the product of the position dilution of precision (PDOP) and a composite user-equivalent range error (UERE). However, a more appropriate indication of the localization performance is the Cramér-Rao bound (CRB), which is a bound for unbiased estimators. Therefore, the CRB based on (1) for TOAs with a distance bias is derived in Appendix 4. Since the TDOAs in Section 2.3 are calculated by making differences of the TOAs in (1), the CRB based on these TDOAs is the same as the one based on (1).

\section{Localization based on TDOA measurements 3.1. System model}

Let us now focus on TDOA measurements. In passive sensor array or microphone array localization, TDOA measurements are obtained directly by cross-correlating a pair of received signals. Thus, no correlation template is needed, and the clock-offset can be canceled out immediately. We reemphasize that these TDOA measurements are different from the TDOAs calculated by subtracting the TOAs. The data model for these TDOA measurements is given by [31] 
Table 1 LS estimators based on TOAs for locating an asynchronous target

\begin{tabular}{|c|c|c|c|c|c|}
\hline & REFF-USR-LS & REFB-USR-LS & REFF-USRD-LS & REFB-USRD-LS(1) & REFB-USRD-LS(2) \\
\hline Relations & & \multicolumn{4}{|c|}{$\begin{array}{l}\text { The REFF-USR-LS and the REFF-USRD-LS estimate are identical The REFB-USR-LS and the REFB } \\
\text { USRD-LS(2) estimate are identical }\end{array}$} \\
\hline No. of references & 0 & 2 & 0 & 1 & 2 \\
\hline Reference dependency & No & Yes & No & Yes & Yes \\
\hline Literature & Proposed & {$[5,17,18]$} & Proposed & {$[7,22]$} & {$[15]$} \\
\hline Min. no. of anchors, $\mathbf{x}$ of length / & & & $1+2$ & & \\
\hline
\end{tabular}

$$
r_{i, j}=d_{j}-d_{i}+n_{i, j}, \quad i, j \in\{1,2, \ldots, M\}, \quad i \neq j,
$$

where $r_{i, j}$ is the TDOA measurement, which is obtained by cross-correlating the received signal from the $j$ th anchor with the one from the $i$ th anchor. Note that the stochastic properties of the noise terms $n_{i, j}$ are totally different from the ones of the noise terms $n_{i}$ of (1). We approximate $n_{i, j}$ as zero-mean random variables, where $\operatorname{cov}\left(n_{\mathrm{i}, \mathrm{j}}, n_{p, q}\right)=E\left[\left(n_{i, j}-E\left[n_{i, j}\right]\right)\left(n_{p, q}-E\left[n_{p, q}\right]\right)\right]=E$ $\left[n_{i, j} n_{p, q}\right], i, j, p, q, \in\{1,2, \ldots, M\}, i \neq j$, and $p \neq q$. Defining $\mathbf{r}_{i}$ as the collection of the corresponding distances to the $M-1$ TDOA measurements using the $i$ th anchor as a reference, $\mathbf{r}_{i}=\left[r_{i, 1}, \ldots, r_{i, i-1}, r_{i, i+1}, \ldots, r_{i, M}\right]^{T}$, and $\mathbf{n}_{i}=$ $\left[n_{i, 1}, \ldots, n_{i, i-1}, n_{i, i}+1, \ldots, n_{i, M}\right]^{T}$ as the related noise vector, we write (39) in vector form as

$$
\mathbf{r}_{i}=\mathbf{T}_{i 1} \mathbf{d}-d_{i} \mathbf{1}_{M-1}+\mathbf{n}_{i}
$$

Moving $-d_{i} \mathbf{1}_{M-1}$ to the other side, making an elementwise multiplication and re-arranging, we achieve

$$
\boldsymbol{\varphi}_{i}=2 \mathbf{T}_{i} \mathbf{X}_{a}^{T} \mathbf{X}+2 d_{i} \mathbf{r}_{i}+\mathbf{m}_{i}
$$

where $\phi_{\boldsymbol{i}}=\mathbf{T}_{\boldsymbol{i}} \boldsymbol{\psi}_{\boldsymbol{a}^{-}} \mathbf{r}_{i} \odot \mathbf{r}_{i}$ and $\mathbf{m}_{i}=-\left(2\left(\mathbf{T}_{i 1} \mathbf{d}\right) \odot \mathbf{n}_{i}+\mathbf{n}\right.$ ${ }_{i} \odot \mathbf{n}_{i}$ ). The stochastic properties of $\mathbf{m}_{i}$ are as follows

$$
\begin{aligned}
E\left[\left[\mathbf{m}_{i}\right]_{k}\right] & =-E\left[\left[\mathbf{n}_{i}\right]_{k} \odot\left[\mathbf{n}_{i}\right]_{k}\right] \approx 0, \\
{\left[\sum_{i}\right]_{k, l}=} & E\left[\left[\mathbf{m}_{i}\right]_{k}\left[\mathbf{m}_{i}\right]_{l}\right]-E\left[\left[\mathbf{m}_{i}\right]_{k}\right] E\left[\left[\mathbf{m}_{i}\right]_{l}\right] \\
& \approx\left\{\begin{array}{l}
4 d_{k} d_{l} E\left[n_{i, k} n_{i, l}\right], \quad k<i \text { and } l<i \\
4 d_{k+1} d_{l+1} E\left[n_{i, k+1} n_{i, l+1}\right], \quad k \geq i \text { and } l \geq i \\
4 d_{k} d_{l+1} E\left[n_{i, k} n_{i, l+1}\right], \quad k<i \text { and } l \geq i \\
4 d_{k+1} d_{l} E\left[n_{i, k+1} n_{i, l}\right], \quad k \geq i \text { and } l<i
\end{array}\right.
\end{aligned}
$$

where we ignore the higher order noise terms to obtain (43) and assume that the noise mean $E\left[[\mathbf{m}]_{i}\right] \approx 0$ under the condition of sufficiently small measurement errors. Note that the noise covariance matrix $\boldsymbol{\Sigma}_{i}$ of size $(M-1) \times(M-1)$ depends on the unknown $\mathbf{d}$ as well.

\subsection{Localization based on squared TDOA measurements}

We do not propose any new algorithms in this section, but summarize existing localization algorithms spread over different research areas and shed light on their relations. All these algorithms are categorized as reference-based SRD approaches. Note that (41) looks similar to (32). Only the available data and the noise characteristics are different, which leads to totally different relations among the estimators as we will show in the following paragraphs. The approach to achieve the REFB-USRD-LS(1) estimate, the REFB-USRD-LS(2) estimate and the REFB-SRD-CWLS estimate (36) based on TOA measurements in Section 2.3.2 can be adopted here as well. The orthogonal projection $\widetilde{\mathbf{P}}_{i}$ of size $(M-$ $1) \times(M-1)$ onto the complement of $\mathbf{r}_{i}$ is employed, which is given by (16), where we replace $\mathbf{I}_{M}$ and $\mathbf{P u}$ with $\mathbf{I}_{M-1}$ and $\mathbf{r}_{i}$. Let us define the nullspace $\mathcal{N}\left(\tilde{\mathbf{U}}_{i}^{T}\right)=\operatorname{span}\left(\mathbf{r}_{i}\right), \quad$ and $\quad \mathcal{R}\left(\tilde{\mathbf{U}}_{i}\right) \oplus \mathcal{N}\left(\tilde{\mathbf{U}}_{i}^{T}\right)=\mathbb{R}^{M-1}$. Therefore, $\widetilde{\mathbf{P}}_{i}$ is the projection onto $\mathcal{R}\left(\widetilde{\mathbf{U}}_{i}\right)$. As a result, the REFB-USRD-LS(1) and REFB-USRD-WLS(1) esti-

\begin{tabular}{|c|c|c|c|c|c|}
\hline & REFF-USR-WLS & REFB-USR-WLS & REFF-USRD-WLS & REFB-USRD-WLS(1) & REFB-USRD-WLS(2) \\
\hline Relations & & \multicolumn{4}{|c|}{$\begin{array}{l}\text { The REFB-USR-WLS and the REFB-USRD-WLS(2) estimate are identical They are all identical with } \\
\text { optimal weighting matrices } \mathbf{Q}^{*}=\mathbf{Q}_{i, j}^{*}=\mathbf{Q}_{i}^{*}\end{array}$} \\
\hline No. of references & 0 & 2 & 0 & 1 & 2 \\
\hline Reference dependency & No & $\begin{array}{l}\text { Yes, with } \mathbf{Q}_{i, j} \\
\text { No, with } \mathbf{Q}_{i, j}^{*}\end{array}$ & No & $\begin{array}{l}\text { Yes, with } \mathbf{Q}_{i} \\
\text { No, with } \mathbf{Q}_{i}^{*}\end{array}$ & $\begin{array}{l}\text { Yes, with } \mathbf{Q}_{\mathrm{i}, j} \\
\text { No, with } \mathbf{Q}_{i, j}^{*}\end{array}$ \\
\hline Literature & Proposed & & Proposed & & \\
\hline Min. no. of anchors, $\mathbf{x}$ of length / & & & $1+2$ & & \\
\hline
\end{tabular}
mate based on TDOA measurements is respectively given by,

$$
\hat{\mathbf{x}}=-\frac{1}{2}\left(\mathbf{X}_{a} \mathbf{T}_{i}^{T} \widetilde{\mathbf{P}}_{i} \mathbf{T}_{i} \mathbf{X}_{a}^{T}\right)^{-1} \mathbf{X}_{a} \mathbf{T}_{i}^{T} \widetilde{\mathbf{P}}_{i} \varphi_{i}
$$

Table 2 WLS estimators based on TOAs for locating an asynchronous target 
Table 3 CLS estimators based on TOAs for locating an asynchronous target

\begin{tabular}{llll}
\hline & REFF-SR-CWLS & REFF-SRD-CWLS & REFB-SRD-CWLS \\
\hline Equations & & $(14)$ & $(36)$ \\
No. of references & 0 & 0 & 1 \\
Reference dependency & No & No & Yes, with $\mathbf{W}_{i}$ \\
& Proposed & Proposed with $\mathbf{W}_{i}^{*}$ \\
Literature & & I+2 & [26] \\
Min. no. of anchors, $\mathbf{x}$ of length / &
\end{tabular}

and

$$
\hat{\mathbf{x}}=-\frac{1}{2}\left(\mathbf{X}_{a} \mathbf{T}_{i}^{T} \widetilde{\mathbf{Q}}_{i} \mathbf{T}_{i} \mathbf{X}_{a}^{T}\right)^{-1} \mathbf{X}_{a} \mathbf{T}_{i}^{T} \widetilde{\mathbf{Q}}_{i} \varphi_{i}
$$

where $\widetilde{\mathbf{Q}}_{i}$ is an aggregate weighting matrix of size $(M$ $1) \times(M-1)$ as well. Note that (44) (or (45)) differs from (18) (or (19)) since $M-1$ TDOA measurements are used instead of $M$ TOA measurements. The optimal $\widetilde{\mathbf{Q}}_{i}$ is given by

$$
\begin{aligned}
& \widetilde{\mathbf{Q}}_{i}^{*}=\widetilde{\mathbf{P}}_{i}\left(\widetilde{\mathbf{P}}_{i} \boldsymbol{\Sigma}_{i} \widetilde{\mathbf{P}}_{i}\right)^{\dagger} \widetilde{\mathbf{P}}_{i} \\
& =\left(\widetilde{\mathbf{P}}_{i} \boldsymbol{\Sigma}_{i} \widetilde{\mathbf{P}}_{i}\right)^{\dagger},
\end{aligned}
$$

where $\widetilde{\mathbf{Q}}_{i}^{*}, i \in\{1, \ldots, M\}$ is the pseudo-inverse of the matrix achieved by projecting the columns and rows of $\boldsymbol{\Sigma}_{i}$ onto $\mathcal{R}\left(\widetilde{\mathbf{U}}_{i}\right)$, which is of rank $M-2$. We remark that the REFB-USRD-LS(1) estimate (44) is equivalent to the ones in $[22,32]$.

Let us also revisit the REFB-USRD-LS(2) estimate and the REFB-USRD-WLS(2) estimate based on TDOA measurements. A linear transformation $\widetilde{\mathbf{M}}_{j}$ of size $(M-2) \times$ $(M-1)$, which fulfills $\widetilde{\mathbf{M}}_{j} \mathbf{r}_{i}=\mathbf{0}_{M-2}$, can be devised in the same way as $\mathbf{M}_{j}$ by replacing $\mathbf{T}_{i} \mathbf{u}$ and $1 /\left(u_{j}-u_{i}\right)$ with $\mathbf{r}_{i}$ and $1 / r_{i, j}$, respectively. Thus, $\mathcal{R}\left(\tilde{\mathbf{M}}_{j}^{T}\right)=\mathcal{R}\left(\widetilde{\mathbf{U}}_{i}\right)$. Note that another heuristic method to obtain $\widetilde{\mathbf{M}}_{j}$ is proposed in [20]. As a result, the general form of the REFB-USRD-LS (2) and the REFB-USRD-WLS(2) estimates can be derived in the same way as (44) and (45) by replacing $\widetilde{\mathbf{P}}_{i}$ and $\widetilde{\mathbf{Q}}_{i}$ with $\widetilde{\mathbf{M}}_{j}^{T} \widetilde{\mathbf{M}}_{j}$ and $\widetilde{\mathbf{Q}}_{i, j}$, respectively. Note that $\widetilde{\mathbf{Q}}_{i, j}$ is also an aggregate weighting matrix of size $(M-1) \times(M-1)$. The optimal $\widetilde{\mathbf{Q}}_{i, j}^{*}$ is given by

$$
\begin{aligned}
& \widetilde{\mathbf{Q}}_{i, j}^{*}=\widetilde{\mathbf{M}}_{j}^{T}\left(\tilde{\mathbf{M}}_{j} \Sigma_{i} \tilde{\mathbf{M}}_{j}^{T}\right)^{-1} \widetilde{\mathbf{M}}_{j} \\
& =\left[\left(\tilde{\mathbf{M}}_{j}\right)^{\dagger} \widetilde{\mathbf{M}}_{j} \Sigma_{i} \tilde{\mathbf{M}}_{j}^{T}\left(\tilde{\mathbf{M}}_{j}^{T}\right)^{\dagger}\right]^{\dagger},
\end{aligned}
$$

where $\left(\widetilde{\mathbf{M}}_{j}\right)^{\dagger} \tilde{\mathbf{M}}_{j}=\widetilde{\mathbf{M}}_{j}^{T}\left(\tilde{\mathbf{M}}_{j}^{T}\right)^{\dagger}=\widetilde{\mathbf{M}}_{j}^{T}\left(\widetilde{\mathbf{M}}_{j} \tilde{\mathbf{M}}_{j}^{T}\right)^{-1} \widetilde{\mathbf{M}}_{j}$ is also the projection onto $\mathcal{R}\left(\widetilde{\mathbf{U}}_{i}\right)$, which means that $\widetilde{\mathbf{Q}}_{i, j}^{*}=\widetilde{\mathbf{Q}}_{i}^{*}, i, j \in\{1, \ldots, M\}$ and $i \neq j$. The REFB-USRDLS(2) estimate and the REFB-USRD-WLS(2) estimate based on TDOA measurements are generalizations of the estimators proposed in [20]. However, the noise covariance matrix in [20] is a diagonal matrix, and the noise covariance matrix $\boldsymbol{\Sigma}_{i}$ here is a full matrix.

We remark here that with the optimal weighting matrix, the REFB-USRD-WLS(1) estimate (45) and the REFB-USRD-WLS(2) estimate based on the same set of TDOA measurements are identical. However, the optimal weighting matrix cannot decouple the reference dependency. The performance of all the estimates still depends on the reference selection, since the reference dependency is an inherent property of the available measurement data. To further improve the localization performance, the REFB-SRD-CWLS estimate based on (41) can be derived in the same way as the estimate (36) by replacing $\varrho_{i}$ and $\mathbf{B}_{i}$ with $\phi_{i}$ and $2\left[\mathbf{T}_{i} \mathbf{X}_{a}^{T}, \mathbf{r}_{i}\right]$, respectively. A solution to this CLS problem is presented in [26].

Note that all the above estimators are based on a socalled nonredundant set of TDOA measurements [31], resulting in reference dependency. Recently, a SM method based on the full set of TDOA measurements has been proposed in [33], labeled "reference-free TDOA source localization". It is reference-free in the sense that every anchor plays the role of reference, as in [17], thus there is no need to specifically choose one. We revisit the proposed method in [33] here to clarify its relation to our framework. Let us define $\mathbf{D}_{r}=\left[\widetilde{\mathbf{r}}_{1}, \widetilde{\mathbf{r}}_{1}, \ldots, \widetilde{\mathbf{r}}_{M}\right]$, where $\widetilde{\mathbf{r}}_{i}$ can be achieved by inserting a 0 in $\mathbf{r}_{i}$ between $r_{i, i-1}$ and $r_{i, i+1}$. Using our notations, we can rewrite (22) of [33] as

$$
\frac{1}{2 M}\left(\mathbf{D}_{r} \odot \mathbf{D}_{r}\right) \mathbf{1}_{M}-\frac{1}{M} \mathbf{D}_{r} \mathbf{d}=\frac{1}{2} \mathbf{P} \psi_{a}-\mathbf{P} \mathbf{X}_{a}^{T} \mathbf{x} .
$$

Then, a matrix $\mathbf{G}$ of size $(M-2) \times M$, which fulfills $\mathbf{G D}_{r}=\mathbf{0}_{M-2}$, can be obtained by exploring the nullspace 
of $\mathbf{D}_{r}$ using the singular value decomposition (SVD). Consequently, an LS estimator of $\mathbf{x}$ is given by

$$
\hat{\mathbf{x}}=\frac{1}{2}\left(\mathbf{X}_{a} \mathbf{P G}^{T} \mathbf{G P X} \mathbf{X}_{a}^{T}\right)^{-1} \mathbf{X}_{a} \mathbf{P G} \mathbf{G}^{T} \mathbf{G}\left(\mathbf{P} \psi_{a}-\frac{1}{M}\left(\mathbf{D}_{r} \odot \mathbf{D}_{r}\right) \mathbf{1}_{M}\right) .
$$

Note that $\mathbf{D}_{r}=\left[\mathbf{d}, \mathbf{1}_{M}\right]\left[\begin{array}{c}\mathbf{1}_{M}^{T} \\ -\mathbf{d}^{T}\end{array}\right]$ without noise, and $\mathbf{G D}_{r}=\mathbf{0}_{M-2}$. Thus, $\mathbf{1}_{M}$ is in the nullspace of $\mathbf{G}$. As $\mathbf{P}$ is the projection onto the orthogonal complement of $\mathbf{1}_{M}$, GP is still of rank $M-2$ with probability 1 . In a different way, we can make use of the full set of TDOA measurements similarly as the second extension of the approach proposed in [32]. We collect (41) in vector form as

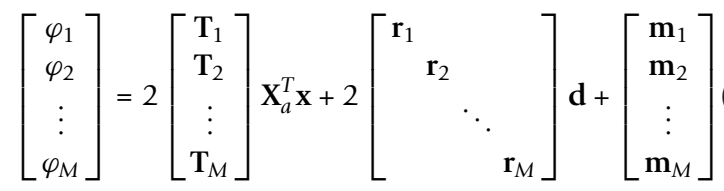

As a result, a LS estimator of $\mathbf{x}$ and $\mathbf{d}$ can be derived based on (52). We do not detail it in the interest of brevity.

Furthermore, as indicated in [31], an optimal nonredundant set can be achieved by the optimum conversion of the full TDOA set in order to approach the same localization performance, and the use of this optimal nonredundant set is recommended to reduce the complexity. Because [31] relies on the assumption that the received signals at the anchors are corrupted by noise with equal variances, the optimal nonredundant set can be estimated by a LS estimator. This is not the case here however, where it should be estimated by a WLS estimator, which requires the knowledge of the stochastic properties of the noise.

We summarize the characteristics of all the estimators based on TDOA measurements in Table 4. With the nonredundant TDOA measurement set of length $M-1$, the estimator performance suffers from a poor reference selection. Although the performance improves with the full set or the optimal nonredundant set, it first has to measure the full set of TDOAs of length $M(M-1) / 2$.

\section{Numerical results}

\subsection{Noise statistics}

In order to make a fair comparison between the localization performance of the different estimators using TOA measurements and TDOA measurements, we derive the statistics of $n_{i}$ and $n_{i, j}$ based on the same received signal models. The received signal is modeled by [33]

$$
z_{i}(n)=\frac{\kappa}{d_{i}} s\left(n-\tau_{i}\right)+e_{i}(n), \quad n=0,1, \ldots, N-1,
$$

where $N$ is the number of samples, $\kappa$ is a constant parameter, $s(n)$ is the source signal, and $e_{i}(n)$ and $\tau_{i}$ are respectively the additive noise and the delay at the $i$ th node. We assume that $s(n)$ is a zero-mean white sequence with variance $\sigma_{s}^{2}$, and $e_{i}(n)$ is also a zeromean white sequence with variance $\sigma_{e}^{2}$, independent from the other noise sequences and $s(n)$.

For the TOA-based approaches, we assume knowledge of the template $s(n)$, and estimate $\tau_{i}$ by cross-correlating the received signal with the clean template:

$$
\hat{\tau}_{i}=\underset{\tau_{i}}{\arg \max }\left\{\sum_{n=0}^{N-1} z_{i}(n) s\left(n-\tau_{i}\right)\right\} .
$$

Since there is an unknown bias due to asynchronous nodes, the distance $u_{i}$ corresponding to the timestamp is modeled as $u_{i}=c \hat{\tau}_{i}=d_{i}+b+n_{i}$, where $c$ is the signal propagation speed. The statistical properties of $n_{i}$ can be derived in a similar way as in [31], and are given by

$$
\begin{aligned}
E\left[n_{i}\right]=0, & \\
\operatorname{Cov}\left(n_{i}, n_{j}\right) & =E\left[n_{i} n_{j}\right] \\
& =\left\{\begin{array}{l}
\sigma_{i}^{2}=\frac{3 c^{2}}{N \pi^{2} \kappa^{2}} \frac{d_{i}^{2}}{\operatorname{SNR}} \begin{array}{l}
i=j, \\
i \neq j
\end{array}
\end{array}\right.
\end{aligned}
$$

\begin{tabular}{|c|c|c|c|c|c|}
\hline & REFB-USRD-LS(1) & REFB-USRD-WLS(1) & REFB-USRD-LS(2) & REFB-USRD-WLS(2) & REFB-SRD-CWLS \\
\hline Relations & \multicolumn{5}{|c|}{$\begin{array}{l}\text { The REFB-USRD-WLS(1) and the REFB-USRD-WLS(2) estimate are identical with the optimal weighting matrices } \\
\widetilde{\mathbf{Q}}_{i}^{*}=\widetilde{\mathbf{Q}}_{i, j}^{*}\end{array}$} \\
\hline No. of references & 1 & 1 & 2 & 2 & 1 \\
\hline Reference dependency & Yes & Yes & Yes & Yes & Yes \\
\hline Literature & {$[19,22,32,33,36]$} & {$[21]$} & [20] & [20] & {$[4,26]$} \\
\hline Min. no. of anchors, $\mathbf{x}$ of length / & & & $1+2$ & & \\
\hline
\end{tabular}

where $\mathrm{SNR}=\sigma_{s}^{2} / \sigma_{e}^{2}$. We remark that in reality, it is very difficult to obtain a clean template, since there are various kinds of error sources, such as multipath fading, antenna mismatch, pulse distortion, etc. Plugging (55)

Table 4 LS, WLS, and CWLS estimators based on TDOAs for locating an asynchronous target 
and (56) into (5), the entries of the covariance matrix $\Sigma$ are given by

$$
\begin{aligned}
{[\Sigma]_{i, j} } & =4 d_{i} d_{j} E\left[n_{i} n_{j}\right]+E\left[n_{i}^{2} n_{j}^{2}\right]-\sigma_{i}^{2} \sigma_{j}^{2} \\
& =\left\{\begin{array}{ll}
4 d_{i}^{2} \sigma_{i}^{2}+2 \sigma_{i}^{4} \approx \frac{12 c^{2}}{N \pi^{2} \kappa^{2}} \frac{d_{i}^{4}}{\mathrm{SNR}^{4}}, i=j \\
0, & i \neq j
\end{array} .\right.
\end{aligned}
$$

On the other hand, the TDOA estimates can be achieved by cross-correlating two received signals as follows

$$
\hat{\tau}_{i, j}=\underset{\tau_{i, j}}{\arg \max }\left\{\sum_{n=0}^{N-1} z_{i}(n) z_{j}\left(n-\tau_{i, j}\right)\right\} .
$$

Thus, the estimate of the distance difference is $r_{i, j}=c \hat{\tau}_{i, j}=d_{j}-d_{i}+n_{i, j}$, where the bias is canceled out naturally. The statistical properties of $n_{i, j}$ can also be derived in a similar way as in $[31,33]$, and are given by

$$
E\left[n_{i, j}\right]=0,
$$

$$
\operatorname{cov}\left(n_{i j}, n_{p, q}\right)= \begin{cases}\frac{3 c^{2}}{N \pi^{2} \kappa^{2}}\left(\frac{d_{i}^{2}}{\operatorname{SNR}}+\frac{d_{j}^{2}}{\operatorname{SNR}}+\frac{d_{i}^{2} d_{j}^{2}}{\mathrm{SNR}^{2}}\right) & i=p \text { and } j=q \\ \frac{3 c^{2}}{N \pi^{2} \kappa^{2}} \frac{d_{i}^{2}}{\operatorname{SNR}} & i=p \text { and } j \neq q \\ \frac{3 c^{2}}{N \pi^{2} \kappa^{2}} \frac{d_{j}^{2}}{\operatorname{SNR}} & j=q \text { and } i \neq p \\ -\frac{3 c^{2}}{N \pi^{2} \kappa^{2}} \frac{d_{i}^{2}}{\operatorname{SNR}} & i=q \text { and } j \neq p \\ -\frac{3 c^{2}}{N \pi^{2} \kappa^{2}} \frac{d_{j}^{2}}{\operatorname{SNR}} & j=p \text { and } i \neq q \\ 0 & \text { else }\end{cases}
$$

Note that similarly as in [33] the signal attenuation is taken into account in order to obtain more general noise statistics than in [31], but we correct the derivation errors in [33]. We remark that in reality, the TDOA estimates may face similar problems as the TOA estimates, since the received signals at different anchors may be totally different. Plugging (59) and (60) into (43), the entries of the covariance matrix $\boldsymbol{\Sigma}_{i}$ are given by

$$
\begin{gathered}
{\left[\sum_{i}\right]_{k, l} \approx \begin{cases}4 d_{k} d_{l} E\left[n_{i, k} n_{i, l}\right], \quad k<i \text { and } l<1 \\
4 d_{k+1} d_{l+1} E\left[n_{i, k+1} n_{i, l+1}\right], \quad k \geq i \text { and } l \geq i \\
4 d_{k} d_{l+1} E\left[n_{i, k} n_{i, l+1}\right], \quad k<i \text { and } l \geq i \\
4 d_{k+1} d_{l} E\left[n_{i, k+1} n_{i, l}\right], & k \geq i \text { and } l<i\end{cases} } \\
= \begin{cases}\frac{12 c^{2} d_{k}^{2}}{N \pi^{2} \kappa^{2}}\left(\frac{d_{i}^{2}}{\mathrm{SNR}^{2}}+\frac{d_{k}^{2}}{\mathrm{SNR}}+\frac{d_{i}^{2} d_{k}^{2}}{\mathrm{SNR}^{2}}\right), & k=l \text { and } k<i \\
\frac{12 c^{2} d_{k+1}^{2}}{N \pi^{2} \kappa^{2}}\left(\frac{d_{i}^{2}}{\mathrm{SNR}^{2}}+\frac{d_{k+1}^{2}}{\mathrm{SNR}}+\frac{d_{i}^{2} d_{k+1}^{2}}{\mathrm{SNR}^{2}}\right), & k=l \text { and } k \geq i \\
\frac{12 c^{2} d_{k} d_{l}}{N \pi^{2} \kappa^{2}} \frac{d_{i}^{2}}{\mathrm{SNR}^{\prime}} & k \neq l, k<i \text { and } l<i \\
\frac{12 c^{2} d_{k+1} d_{l+1}}{N \pi^{2} \kappa^{2}} \frac{d_{i}^{2}}{\mathrm{SNR}^{2}}, & k \neq l, k \geq i \text { and } l \geq i \\
\frac{12 c^{2} d_{k} d_{l+1}}{N \pi^{2} \kappa^{2}} \frac{d_{i}^{2}}{\mathrm{SNR}^{\prime}}, & k<i \text { and } l \geq i \\
\frac{12 c^{2} d_{k+1} d_{l}}{N \pi^{2} \kappa^{2}} \frac{d_{i}^{2}}{\mathrm{SNR}^{2}}, & k \geq i \text { and } l<i\end{cases}
\end{gathered}
$$

In the simulations, we generate $n_{i}$ and $n_{i, j}$ as zeromean Gaussian random variables with covariance matrices specified as above.

\subsection{Performance evaluation}

As a well-adopted lower bound, the CRB is derived for localization estimators based on TOA measurements and TDOA measurements, respectively. Note that the estimators derived in this paper are biased. We remark that although the CRB is a bound for unbiased estimators, it still is interesting to compare it with the proposed biased estimators. Here, we exemplify the CRBs for location estimation on a plane, e.g., we take $l=2$. We assume that $n_{i}$ and $n_{i, j}$ are Gaussian distributed. The Fisher information matrix (FIM) $\mathbf{I}_{1}(\theta)$ based on model (1) in Section 2 for TOA measurements is derived in Appendix 4, where $\boldsymbol{\theta}=\left[\mathbf{x}^{T}, b\right]^{T}$, and $\mathbf{x}=\left[x_{1}\right.$, $\left.x_{2}\right]^{T}$. Consequently, we obtain $\operatorname{CRB}\left(x_{1}\right)=\left[\mathbf{I}_{1}^{-1}(\theta)\right]_{1,1}$. We observe that $b$ is not part of $\mathbf{I}_{1}^{-1}(\theta)$. Therefore, no matter how large $b$ is, it has the same influence on the CRB for TOA measurements. The FIM $\mathbf{I}_{2}(\mathbf{x})$ and $\mathbf{I}_{3}(\mathbf{x})$ based on model (39) in Section 3 are derived in Appendix 5 for the nonredundant set and the full set of TDOA measurements, respectively.

We consider three simulation setups. In Setups 1 and 2 , eight anchors are evenly located on the edges of a $100 \mathrm{~m} \times 100 \mathrm{~m}$ rectangular. Meanwhile the target node is located at $[200 \mathrm{~m}, 30 \mathrm{~m}]$ and $[10 \mathrm{~m}, 20 \mathrm{~m}]$ for Setups 1 and 2, respectively. Thus, the target node is far away from the anchors in Setup 1, but close to them in Setup 2. In Setup 3, all anchors and the target node are randomly distributed on a grid with cells of size $1 \mathrm{~m} \times 1 \mathrm{~m}$ inside the rectangular. The performance criterion is the root mean squared error (RMSE) of $\hat{\mathbf{x}}$ versus a reference range $\left(\mathrm{SNR}_{r}=\frac{N \pi^{2} \kappa^{2}}{3 c^{2}} \mathrm{SNR}\right)$, which can be expressed as $\sqrt{1 / N_{\exp } \sum_{j=1}^{N_{\text {exp }}}\left\|\widehat{\mathbf{x}}^{(j)}-\mathbf{x}\right\|^{2}}$, where $\hat{\mathbf{x}}^{(j)}$ is the estimate obtained in the $j$ th trial. Each simulation result is averaged over $N_{\text {exp }}=1,000$ Monte Carlo trials. The bias $b$ corresponding to the clock offset is randomly generated in the range of $[0 \mathrm{~m}, 100 \mathrm{~m}]$ in each Monte Carlo run. We would like to compare all the REFF and REFB estimators, as well as the estimator proposed in [27] (first iteration) using TOA measurements, labeled the LS1 estimator, and the estimator proposed in [33] using the full TDOA set, namely the REFF-LS2 estimator.

\subsubsection{Estimators using TOA measurements}

Figure 1 shows the localization performance of the REFF estimators using TOA measurements under the three considered setups. The $\mathbf{I}_{1}^{-1}(\theta)$ (the dotted line with " $x$ " 


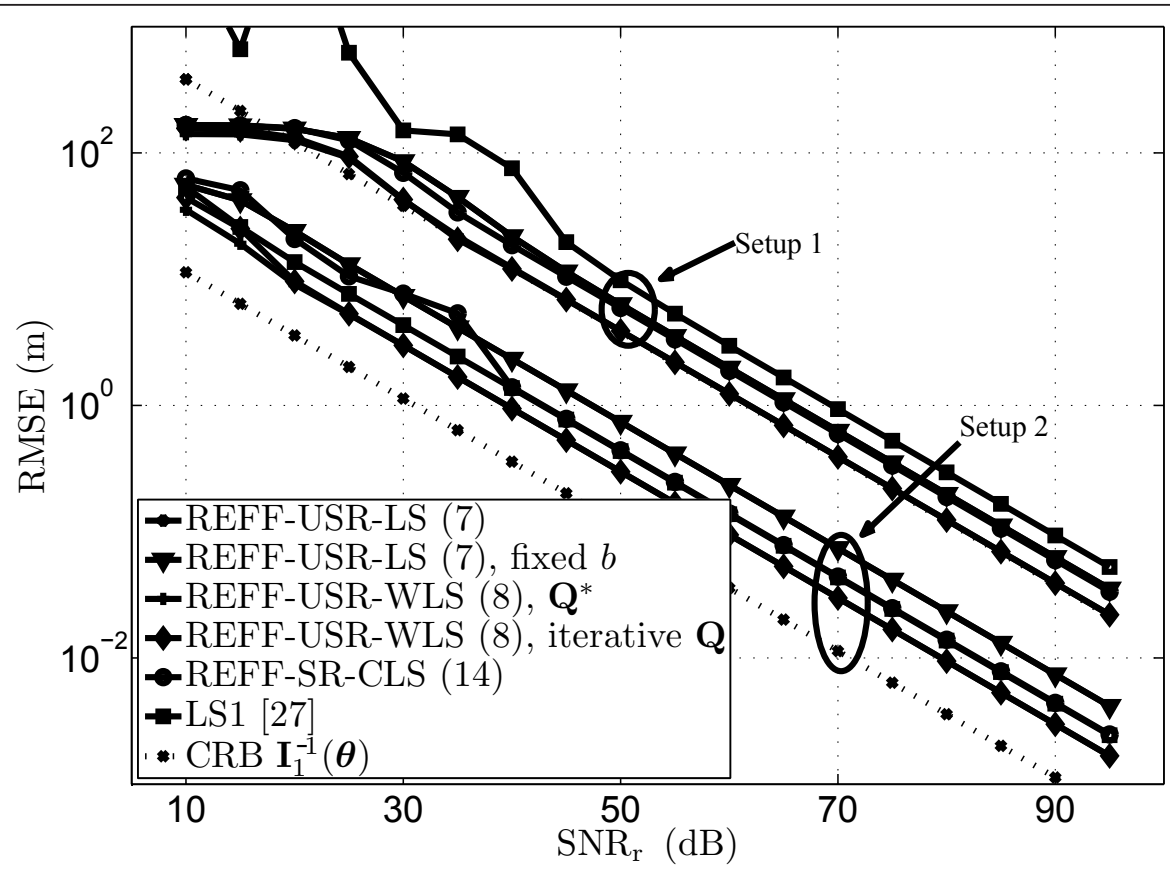

(a)

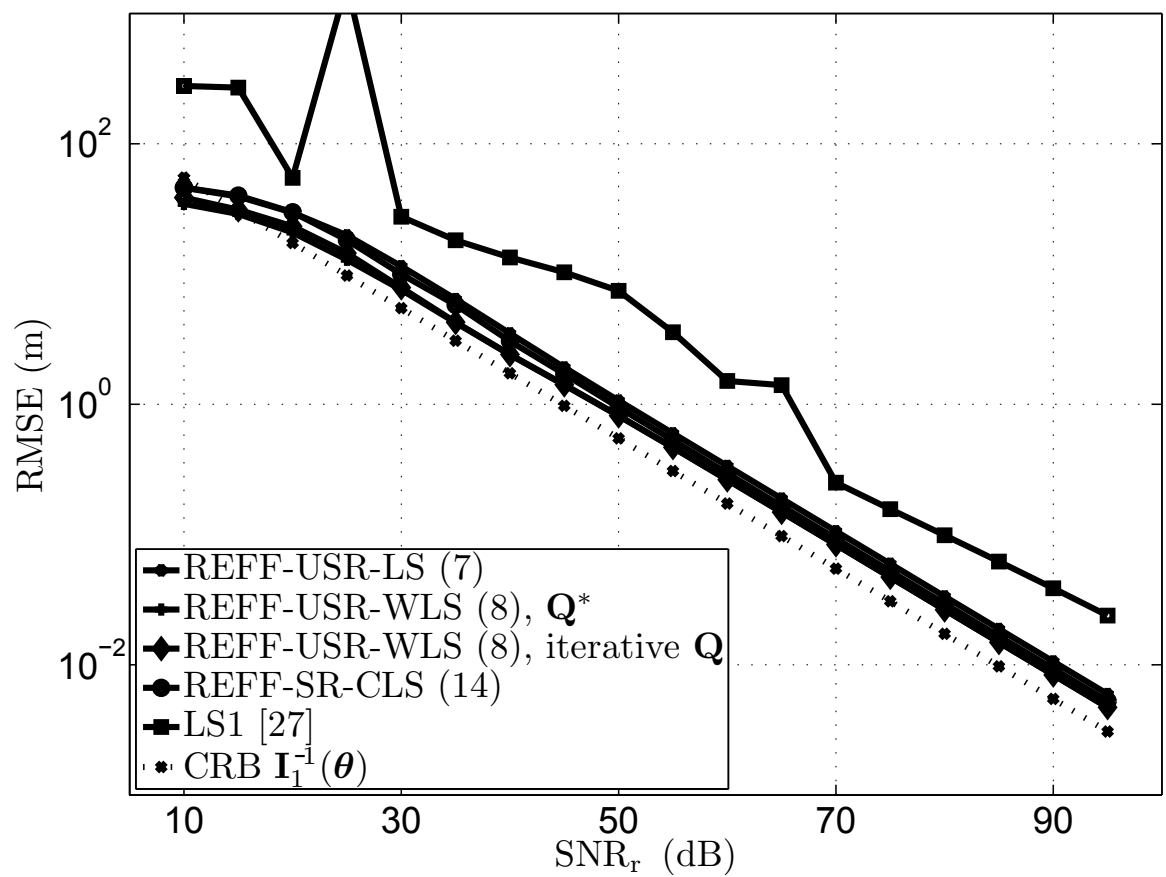

(b)

Figure 1 RMSE of $\mathbf{x}$ for the REFF estimators using TOAs for locating an asynchronous target. (a) Setup 1 and Setup 2. (b) Setup 3.

markers) is used as a benchmark. The REFF-USR-WLS estimator (8) with the optimal weighting matrix (the solid line with "+" markers) achieves the best performance, while the iterative approach to update the weighting matrix (the solid line with " $\diamond$ " markers) also helps the REFF-USR-WLS estimator to converge to the best performance. The REFF-SR-CLS estimator (14) (the solid line with "O" markers) benefits from the 
constraints, and thus outperforms the REFF-USR-LS estimator (7) (the solid line with ":" markers). The concrete value of the bias $b$ does not influence the localization performance. The curve of the REFF-USR-LS estimator with fixed $b$ (the solid line with " $\nabla$ " markers) and the one with random $b$ overlap. Furthermore, the LS1 estimator [27] (the solid line with “ $\square$ " markers) is sensitive to the geometry. It performs better than the REFF-USR-LS estimator in Setup 2, but worse in Setup 1. This observation is consistent with the one in [19]. In Setup 3 (random geometry), it fails under some cases due to its inherent instability, and performs unsatisfactorily.

Figure 2 compares the localization performance of the REFF with the one of the REFB estimators using TOA measurements under Setups 1 and 2. Since there are no fixed anchors in Setup 3, we skip it in the comparison. We show both the performance of the best and the worst reference selection, which indicates the performance limits of the REFB estimators. The dashed lines with "+" and " $\nabla$ " markers denote the performance bounds for the REFBUSRD-LS(1) and the REFB-USRD-LS(2), respectively. The best reference choice for the REFB-USRD-LS(1) estimator is the reference anchor with the shortest distance to the target node. Meanwhile, we do not observe the best reference pair selection for the REFB-USRD-LS(2) estimator following any rules. The curves for the REFF-USR-LS estimator (7) (the solid line with "*" markers) and the REFFSR-CLS estimator (14) (the solid line with "O" markers) lie inside these limits. Their performances are neither too bad nor too good, but they do not suffer from a poor reference selection. As we have already proved that the optimal weighting matrix can compensate the impact of the reference selection, the curves of all the WLS estimators with optimal weights will overlap. Thus, we do not show the performance of the REFF-USR-WLS estimator again, which is already illustrated in Figure 1.

\subsubsection{Estimators using TDOA measurements}

Let us first compare the CRBs employing different measurements in Figure 3. We observe the same tendency for both Setups 1 and 2. All the CRBs overlap above a specific $\mathrm{SNR}_{\mathrm{r}}$ threshold, which is $55 \mathrm{~dB}$ for Setup 1, and $50 \mathrm{~dB}$ for Setup 2. Below the threshold, the CRB using TOA measurements (the solid line with " $x$ " markers) is lower than the other CRBs. Meanwhile, the CRB using the full TDOA set (the dotted line with " $x$ " markers) is lower than the ones using a nonredundant TDOA set (the dotted lines). The observations are consistent with the ones in [31]. On the other hand, the $\mathrm{SNR}_{\mathrm{r}}$ ranges of interest corresponding to a RMSE smaller than $10^{\circ}=1$ $\mathrm{m}$, are $\mathrm{SNR}_{\mathrm{r}}>60 \mathrm{~dB}$ and $\mathrm{SNR}_{\mathrm{r}}>30 \mathrm{~dB}$ for Setup 1 and Setup 2, respectively. Within this range of interest, there are no differences among the CRBs in Setup 1, and only small differences in Setup 2. Therefore, using different measurements would not cause obvious differences in the CRB at high SNR.

Figure 4 shows the localization performance of the REFF estimators using the full TDOA set under three setups. The CRB $\mathbf{I}_{3}^{-1}(\theta)$ (the dotted line with $\times$ markers) is still used as a benchmark. We observe similar tendencies as in Figure 1. The REFF-WLS estimator based on (52) with the optimal weighting matrix (the solid line with "+" markers) achieves the best performance, while the iterative approach to update the weighting matrix (the solid line with " $\diamond$ " markers) also facilitates the REFF-WLS estimator based on (52) to converge to the best performance. Moreover, the performance of the REFF-LS2 estimator (51) [33] (the solid line with “ $\square$ " markers) is slightly worse than the REFFLS estimator based on (52) (the solid line with "*" markers) in Setup 1. In general, their performances are very close. In Setup 3 (random geometry), they almost overlap with each other.

Figure 5 compares the localization performance of the REFF estimator using the full TDOA set with the one of the REFB estimators using the nonredundant TDOA set under Setups 1 and 2. Since there are no fixed anchors in Setup 3, we again skip it in the comparison. We show both the performance of the best and the worst reference selection, which indicates the performance limits of the REFB estimators. The dashed lines with " + " and " $\nabla$ " markers denote the performance limits for the REFBUSRD-LS(1) (44) and the REFB-USRD-LS(2) estimator, respectively. The best reference choice for the REFBUSRD-LS(1) estimator is again the reference anchor with the shortest distance to the target node, which means we cross-correlate the received signal at the reference anchor with the ones at other anchors in order to achieve a nonredundant set of TDOA measurements. Meanwhile, we do not observe the best reference pair selection for the REFB-USRD-LS(2) estimator following any rules either. The curves for the REFF-LS estimator based on (52) (the solid line with "*:" markers) and the REFF-LS2 estimator (51) [33] (the solid line with “ $\square$ " markers) lie inside these limits. They are very close to the lower limits in Setup 1, and in the middle of the performance band in Setup 2 . The performance band of the REFB-USRD-LS(1) estimator is quite narrow in Setup 2. On the other hand, the performance variation is very obvious for the REFBUSRD-LS(2) estimator.

Finally, we verify the equivalence of the REFB-USRDWLS estimators with the same optimal weighting matrix in Figure 6. As we have discussed before, the optimal weighting matrix can only release the impact of the second reference selection. The first reference selection decides the obtained data set. Therefore, using the same nonredundant set of TDOAs, the curves of the REFB- 


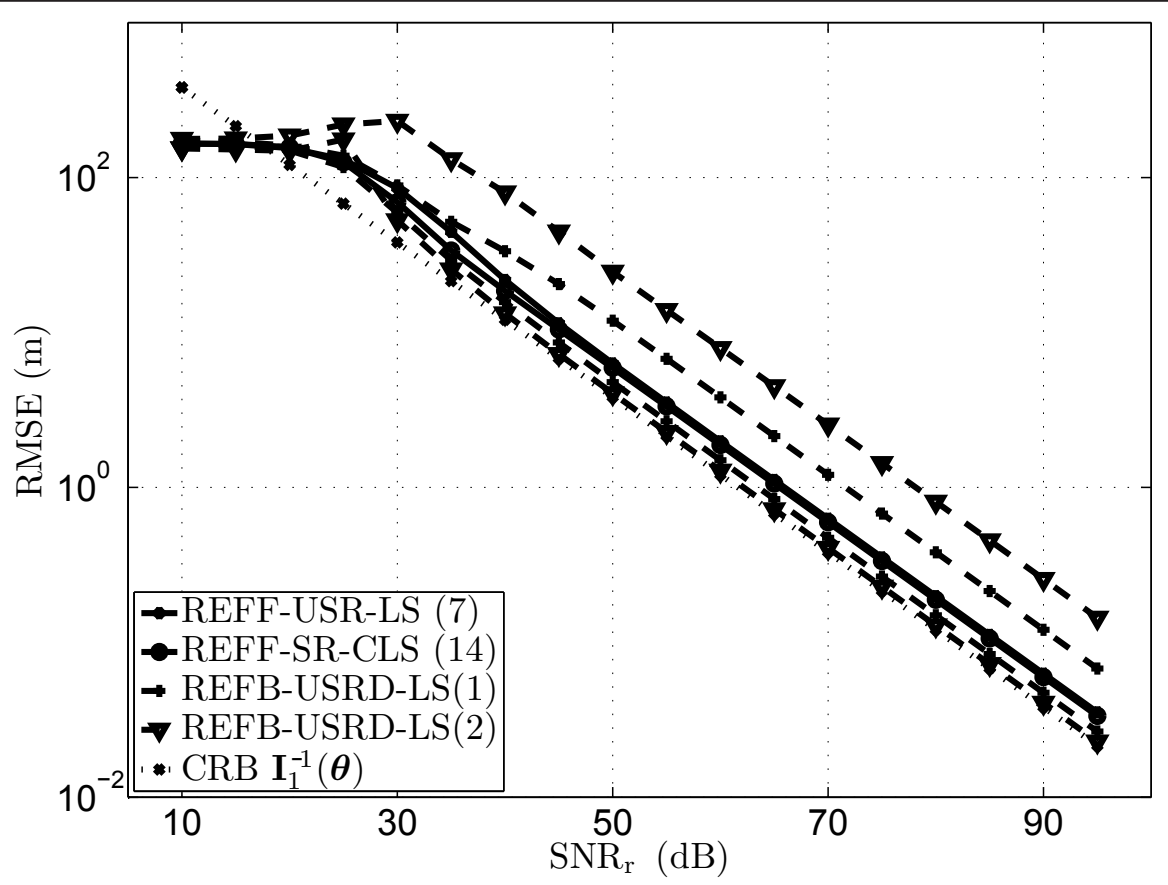

(a)

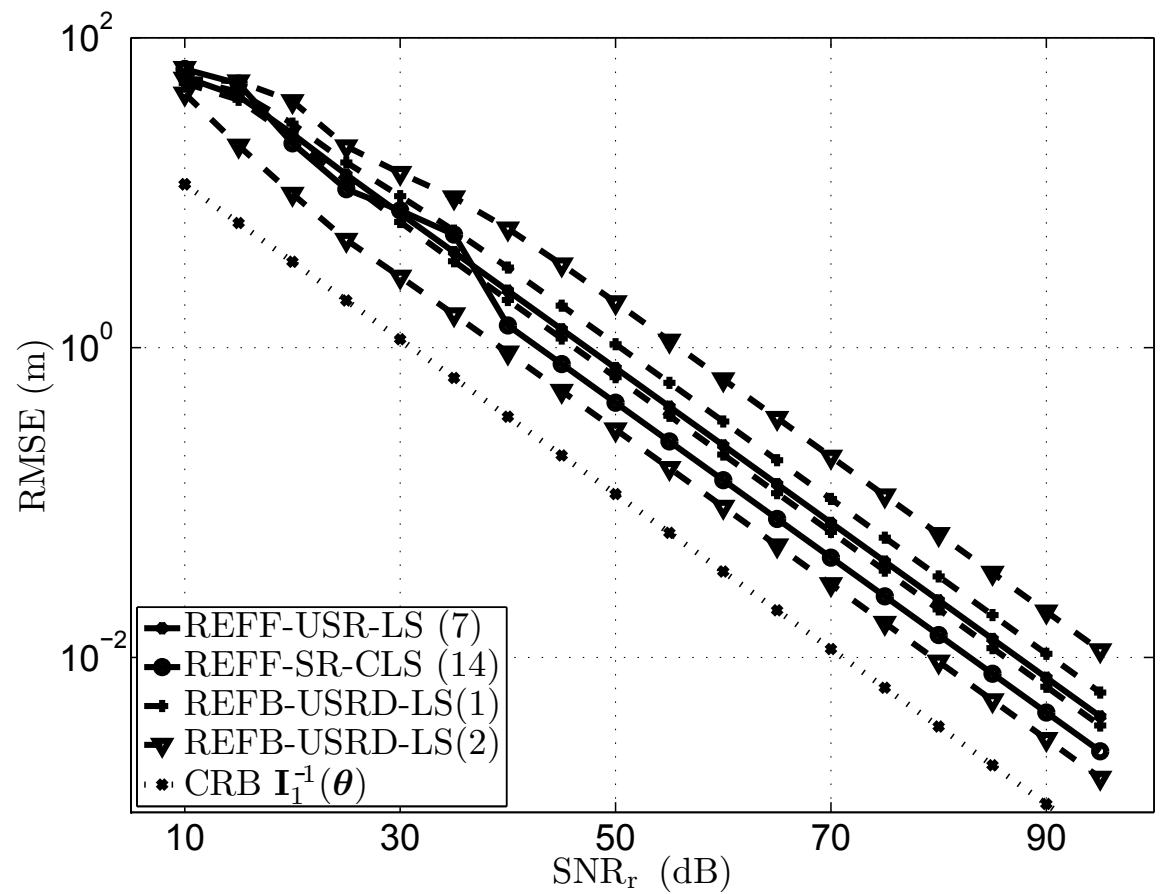

(b)

Figure 2 RMSE of $\mathbf{x}$ for the REFF and the REFB estimators using TOAs for locating an asynchronous target. (a) Setup 1. (b) Setup 2.

USRD-WLS(1) (45) (the solid lines with “ $\diamond$ ” markers) and the REFB-USRD-WLS(2) estimators (the solid lines with "+" markers) overlap. A different performance can be obtained by employing different nonredundant
TDOA sets. However, similarly as the CRB, the performance converges after some $\mathrm{SNR}_{\mathrm{r}}$ threshold. Finally, in Figure 7, we compare the localization performance of the REFF estimators using TOAs and the full TDOA 


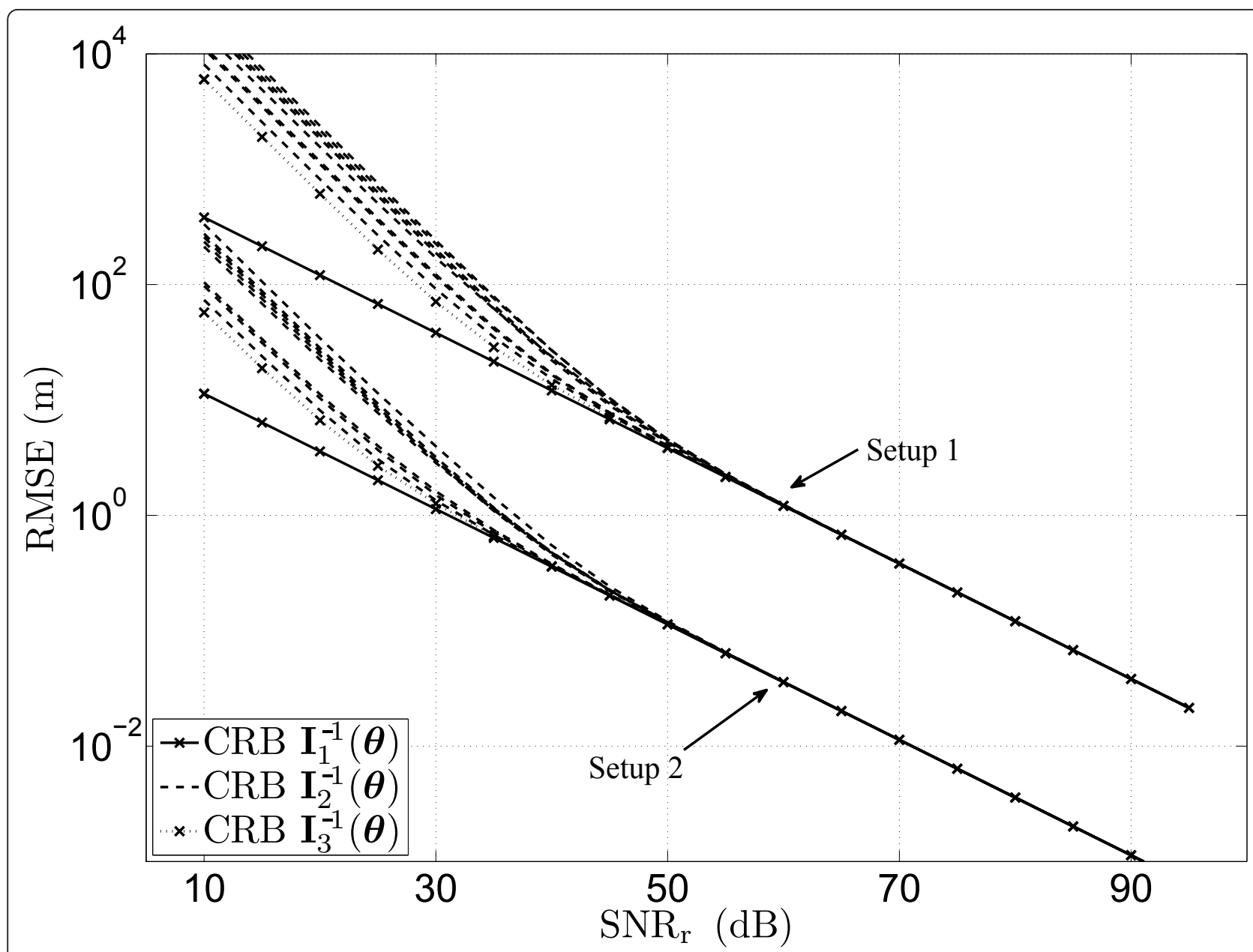

Figure 3 The CRBs using TOAs $\mathbf{I}_{1}^{-1}(\theta)$, the nonredundant TDOA set $\mathbf{I}_{2}^{-1}(\theta)$, and the full TDOA set $\mathbf{I}_{3}^{-1}(\theta)$ for locating an asynchronous target.

set, respectively. They are very close at high $\mathrm{SNR}_{\mathrm{r}}$, but diverge at low $\mathrm{SNR}_{\mathrm{r}}$.

\section{Conclusions}

In this article, we have proposed reference-free localization estimators based on TOA measurements for a scenario, where anchors are synchronized, and the clock of the target node runs freely. The reference-free estimators do not suffer from a poor reference selection, which can seriously degrade the localization performance of reference-based LS estimators. Furthermore, we generalized existing reference-based localization estimators using TOA or TDOA measurements, and expose their relations. Based on analysis and simulations, we have obtained the following important conclusions:

(1) Applying a projection is always preferred over making differences with a reference to get rid of nuisance parameters.
(2) The optimal weighting matrix can compensate for the impact of the reference selection for reference-based WLS estimators using TOA measurements, and make all those estimators equivalent. However, the optimal weighting matrix cannot release the reference influence for reference-based WLS estimators using a nonredundant set of TDOA measurements, but can make the estimators using the same set identical as well.

(3) There are corresponding equivalences between the SR-based and the SR-difference-based methods, which are all using TOA measurements.

(4) Beyond some SNR threshold, there are no obvious differences among the CRBs using TOA measurements, the nonredundant set and the full set of TDOA measurements, respectively.

(5) The performance of the reference-free LS estimators is neither too bad nor too good, but they do not suffer from a poor reference selection. 


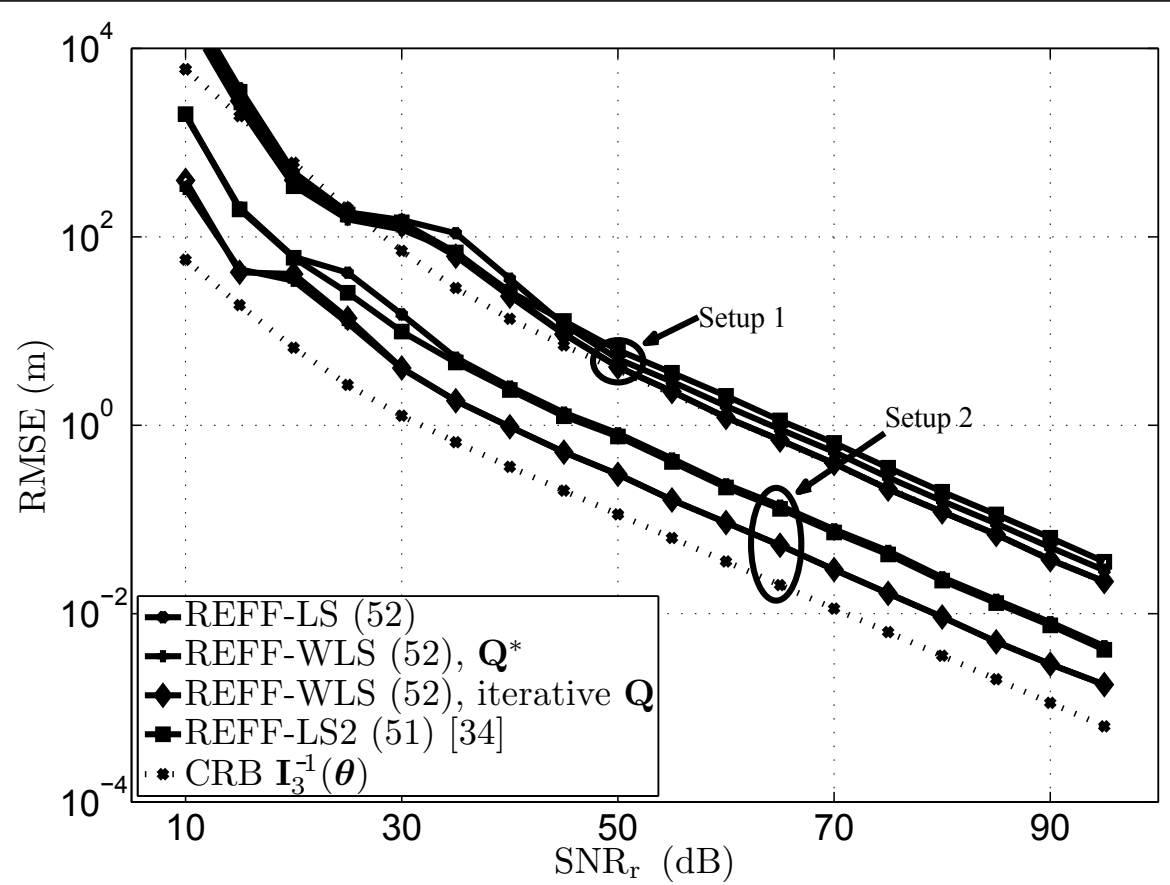

(a)

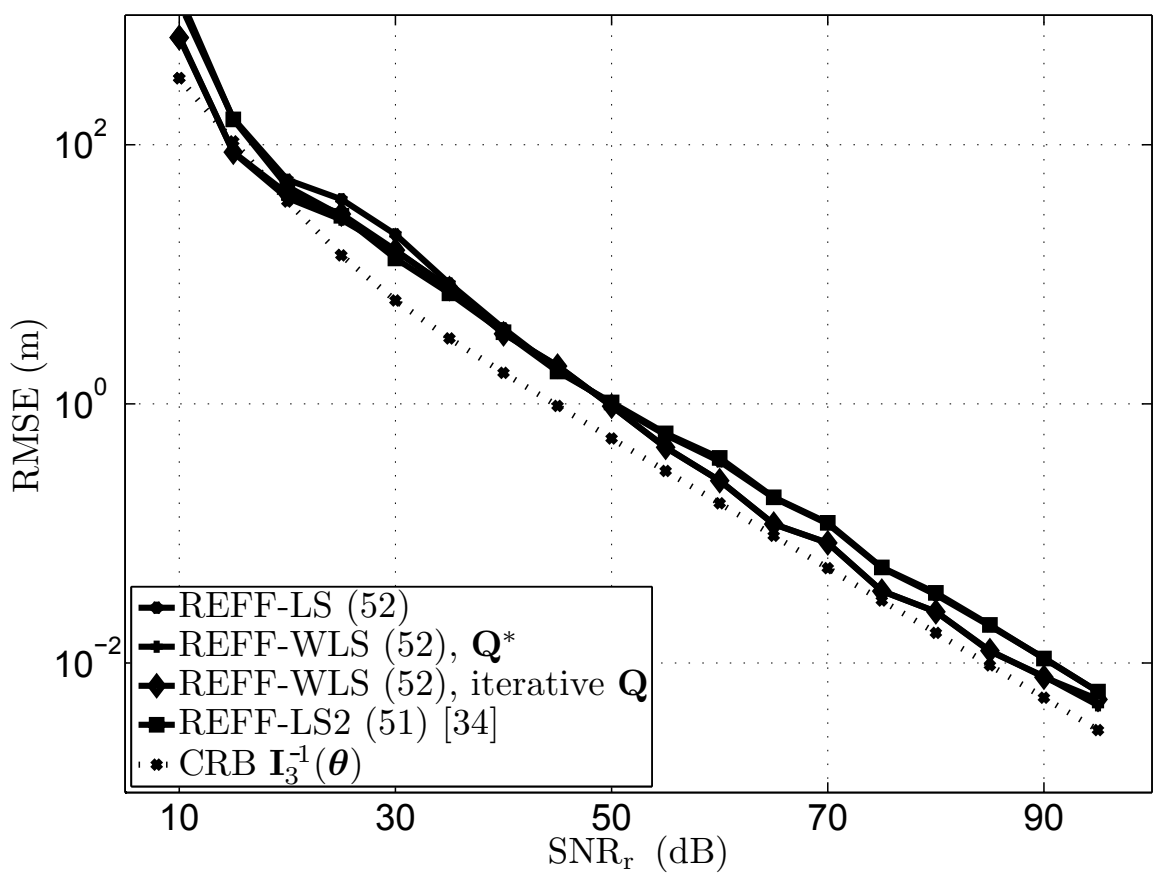

(b)

Figure 4 RMSE of $\mathbf{x}$ for the REFF estimators using the full set of TDOAs for locating an asynchronous target. (a) Setup 1 and Setup 2. (b) Setup 3. 


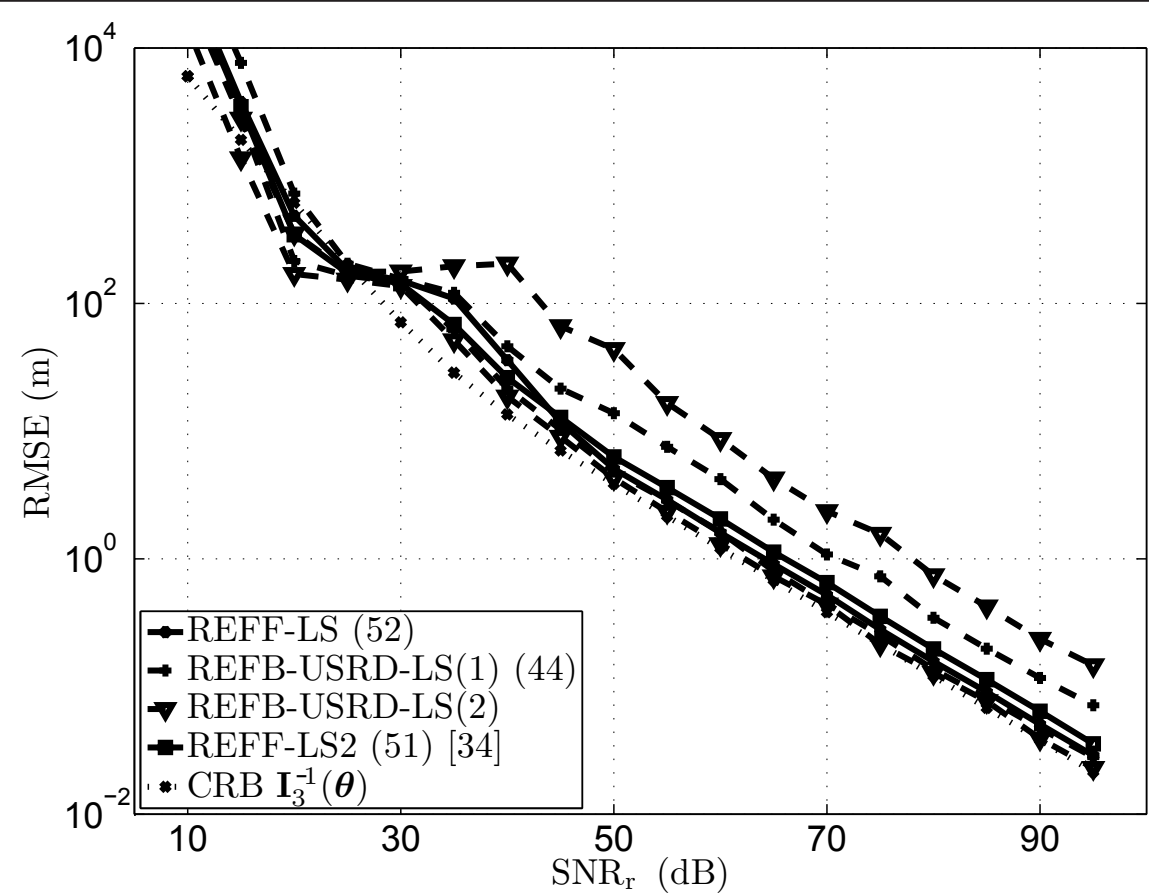

(a)

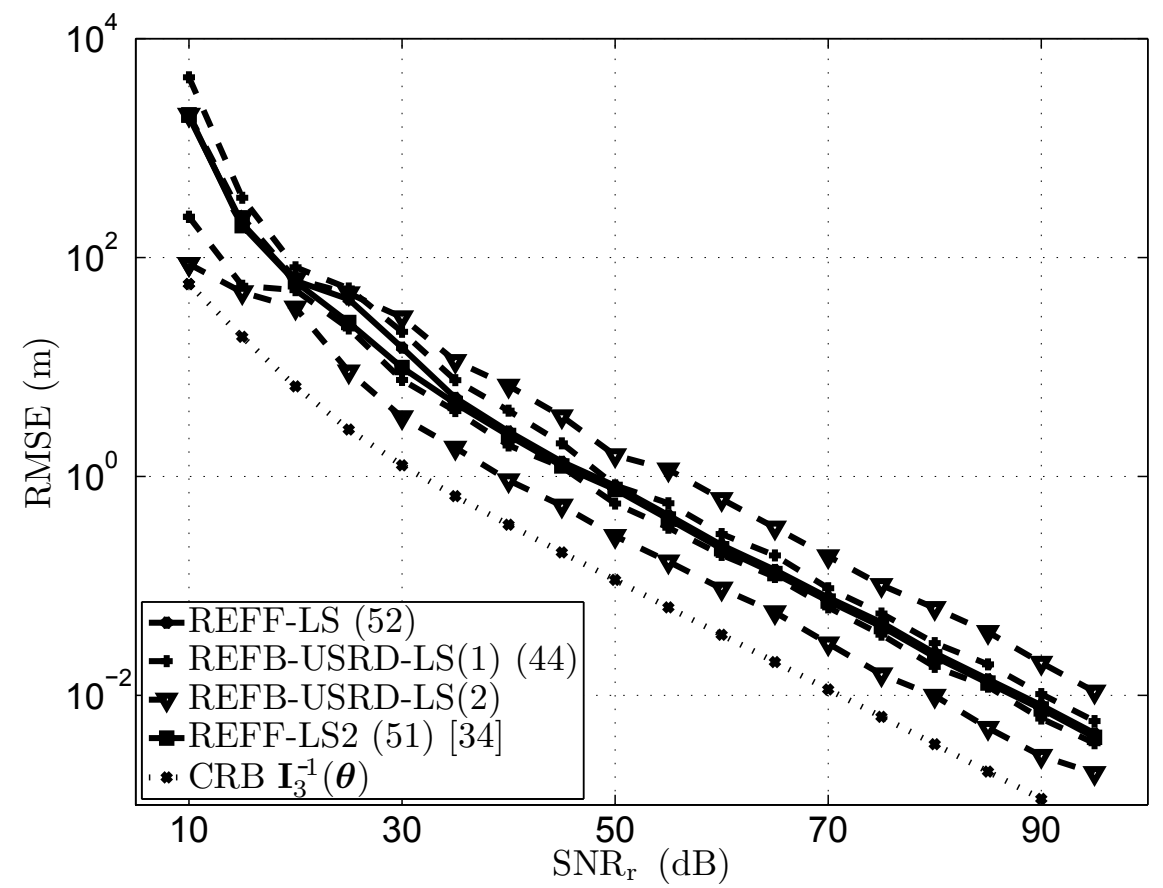

(b)

Figure 5 RMSE of $x$ for the REFF estimator using the full set of TDOAs and the REFB estimators using the nonredundant set of TDOAs for locating an asynchronous target. (a) Setup 1. (b) Setup 2. 


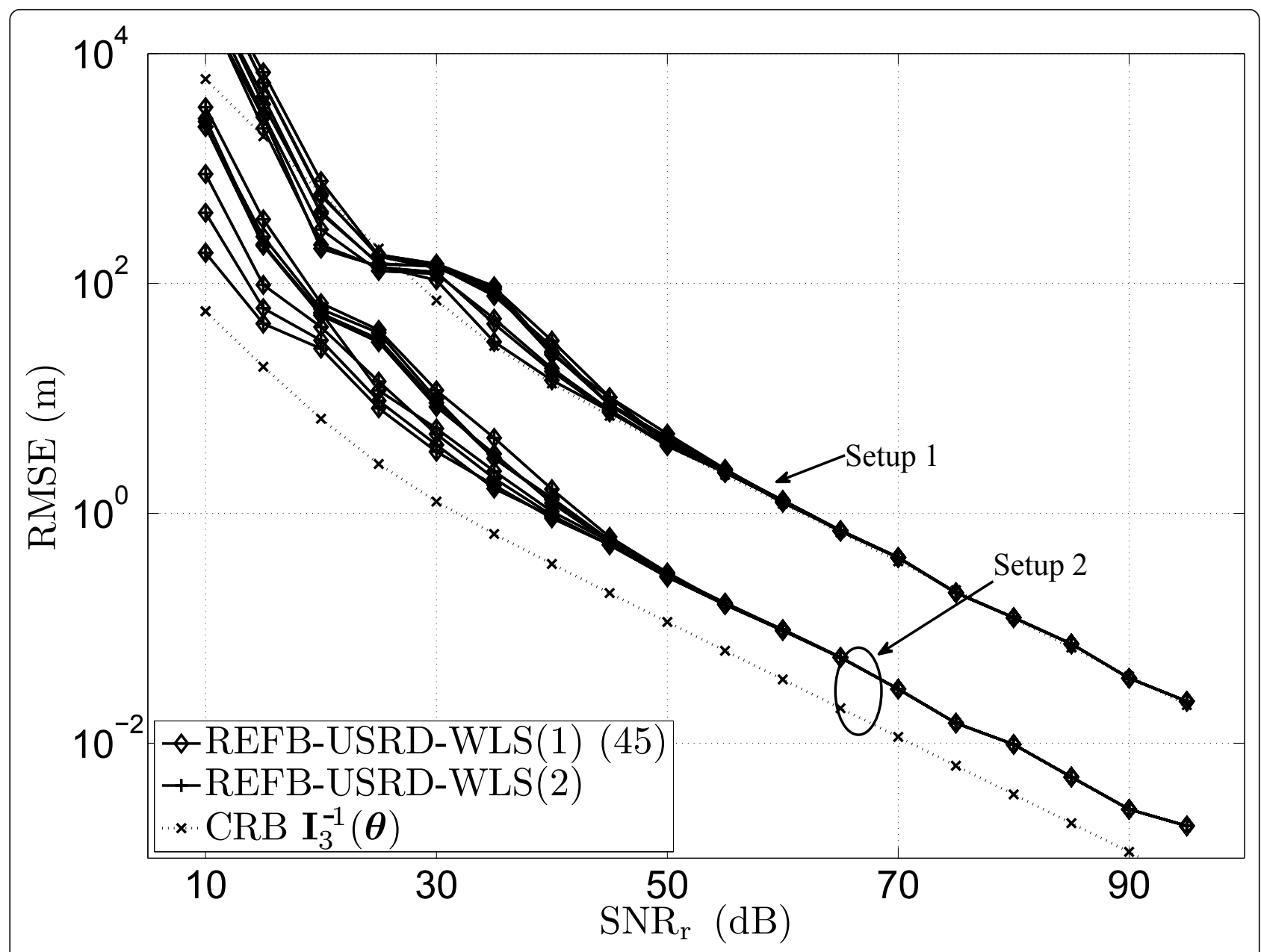

Figure 6 RMSE of $x$ for the REFB-USRD-WLS estimators using the nonredundant set of TDOAs for locating an asynchronous target.

(6) The concrete value of the distance bias caused by the inaccurate clock does not affect the localization performance of the LS or WLS estimators.

\section{Appendix 1 Derivation of $\lambda$ for CLS}

Substituting (14) into the constraint (11), we obtain

$$
\begin{array}{r}
\left(\boldsymbol{\phi}^{T} \mathbf{W}^{T} \mathrm{~A}-\frac{\lambda}{2} \boldsymbol{\rho}^{T}\right)\left(\mathrm{A}^{T} \mathrm{WA}+\lambda \mathrm{J}\right)^{-1} \mathrm{~J}\left(\mathrm{~A}^{T} \mathrm{WA}+\lambda \mathrm{J}\right)^{-1}\left(\mathrm{~A}^{T} \mathrm{~W} \boldsymbol{\phi}-\frac{\lambda}{2} \rho\right) \\
+\boldsymbol{\rho}^{T}\left(\mathrm{~A}^{T} \mathrm{WA}+\lambda \mathrm{J}\right)^{-1}\left(\mathrm{~A}^{T} \mathrm{~W} \boldsymbol{\phi}-\frac{\lambda}{2} \rho\right)=0,
\end{array}
$$

which has to be solved for $\lambda$, leading to the estimate $\hat{\lambda}$. We exemplify how to solve (62) for localization on a plane, i.e., $/=2$. Since $\mathbf{J}$ is of rank 3 , there are only three non-zero eigenvalues of $\left(\mathbf{A}^{T} \mathbf{W A}\right)^{-1} \mathbf{J}$. Therefore, the square matrix $\left(\mathbf{A}^{T} \mathbf{W A}\right)^{-1} \mathbf{J}$ of size $4 \times 4$ can be diagonalized as $\left(\mathbf{A}^{T} \mathbf{W A}\right)^{-1} \mathbf{J}=\mathbf{V} \mathbf{\Lambda} \mathbf{V}^{-1}$, where $\mathbf{V}$ is of size $4 \times 3$, collecting the singular vectors corresponding to the three nonzero singular values, and $\boldsymbol{\Lambda}$ is a diagonal matrix with the three nonzero singular values $\left(\gamma_{i}, i=1\right.$, $2,3)$ on its diagonal. According to the Kailath variant [34] and plugging the eigenvalue decomposition of $\left(\mathbf{A}^{T}\right.$ $\mathbf{W A})^{-1} \mathbf{J}$ into $\left(\mathbf{A}^{T} \mathbf{W A}+\lambda \mathbf{J}\right)^{-1}$, we obtain

$$
\begin{aligned}
\left(\mathbf{A}^{T} \mathbf{W A}+\lambda \mathbf{J}\right)^{-1} & =\left(\mathbf{A}^{T} \mathbf{W A}\right)^{-1}-\lambda\left(\mathbf{A}^{T} \mathbf{W A}\right)^{-1} \mathbf{J}\left(\mathbf{I}+\lambda\left(\mathbf{A}^{T} \mathbf{W A}\right)^{-1} \mathbf{J}\right)^{-1}\left(\mathbf{A}^{T} \mathbf{W A}\right)^{-1} \\
& =\mathbf{V}(\mathbf{I}+\lambda \mathbf{\Lambda})^{-1} \mathbf{V}^{-1}\left(\mathbf{A}^{T} \mathbf{W A}\right)^{-1}
\end{aligned}
$$

Substituting (63) into the constraint (62), we achieve

$$
\begin{aligned}
0 & =\mathrm{e}^{T}(\mathbf{I}+\lambda \boldsymbol{\Lambda})^{-1} \boldsymbol{\Lambda}(\mathbf{I}+\lambda \boldsymbol{\Lambda})^{-1} \mathbf{f}+\frac{\lambda^{2}}{4} \mathbf{h}^{T}(\mathbf{I}+\lambda \boldsymbol{\Lambda})^{-1} \boldsymbol{\Lambda}(\mathbf{I}+\lambda \boldsymbol{\Lambda})^{-1} \mathrm{~g}-\frac{\lambda}{2} \mathbf{h}^{T}(\mathbf{I}+\lambda \boldsymbol{\Lambda})^{-1} \boldsymbol{\Lambda}(\mathbf{I}+\lambda \boldsymbol{\Lambda})^{-1} \mathbf{f} \\
& -\frac{\lambda}{2} \mathrm{e}^{T}(\mathbf{I}+\lambda \boldsymbol{\Lambda})^{-1} \boldsymbol{\Lambda}(\mathbf{I}+\lambda \boldsymbol{\Lambda})^{-1} \mathrm{~g}+\mathbf{h}^{T}(\mathbf{I}+\lambda \boldsymbol{\Lambda})^{-1} \mathbf{f}-\frac{\lambda}{2} \mathbf{h}^{T}(\mathbf{I}+\lambda \boldsymbol{\Lambda})^{-1} \mathrm{~g}
\end{aligned}
$$

where

$$
\begin{aligned}
& \mathbf{e}^{T}=\phi^{T} \mathbf{W}^{T} \mathrm{AV}=\left[\begin{array}{llll}
e_{1} & e_{2} & e_{3} & e_{4}
\end{array}\right], \\
& \mathbf{f}=\mathbf{V}^{-1}\left(\mathbf{A}^{T} \mathbf{W A}\right)^{-1} \mathbf{A}^{T} \mathbf{W} \phi=\left[f_{1} f_{2} f_{3} f_{4}\right]^{T}, \\
& \mathbf{h}^{T}=\rho^{T} \mathbf{V}=\left[\begin{array}{llll}
h_{1} & h_{2} & h_{3} & h_{4}
\end{array}\right],
\end{aligned}
$$




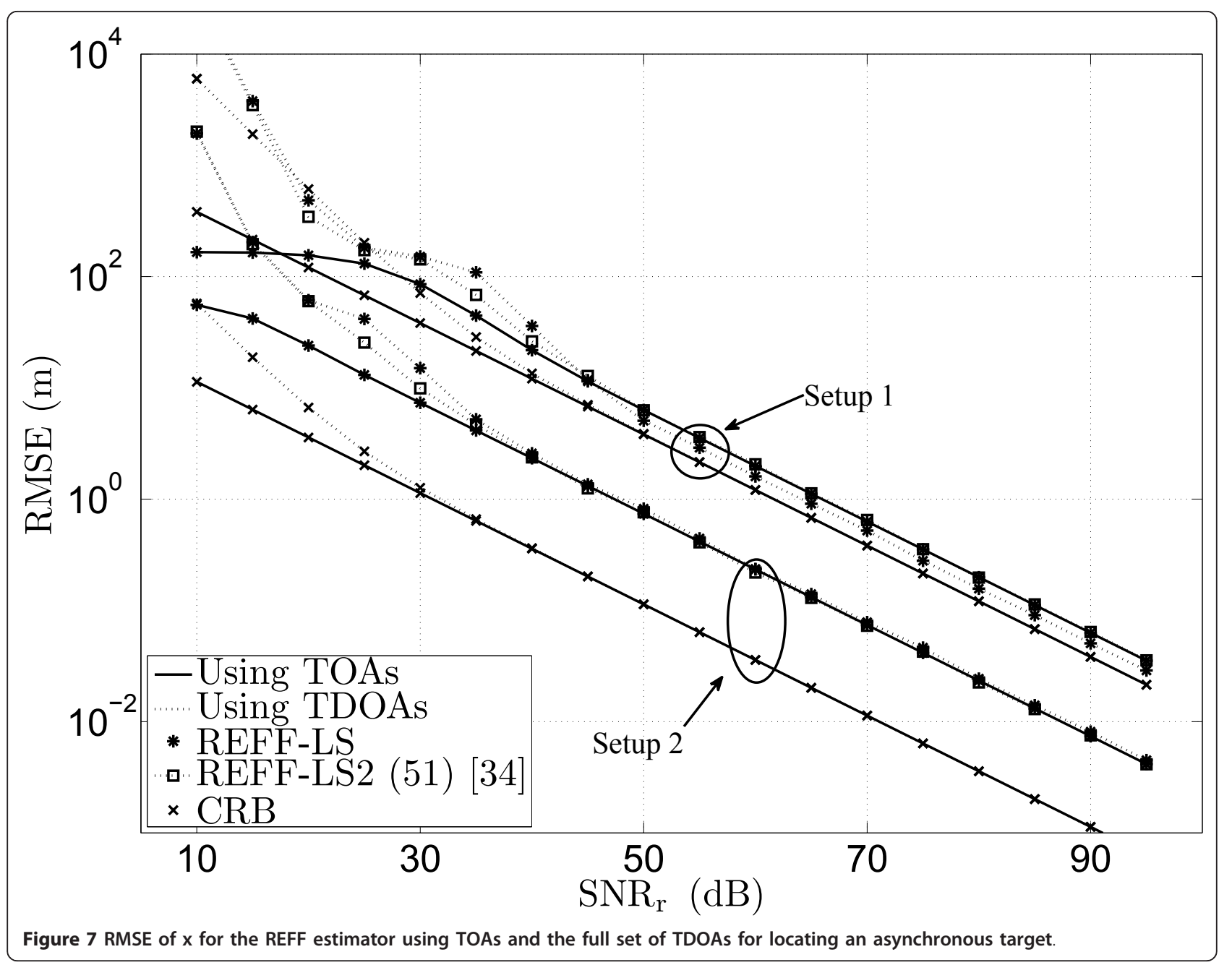

$$
\mathbf{g}=\mathbf{V}^{-1}\left(\mathbf{A}^{T} \mathbf{W A}\right)^{-1} \rho=\left[\begin{array}{llll}
g_{1} & g_{2} & g_{3} & g_{4}
\end{array}\right]^{T} .
$$

Now, (64) can be simplified as a seven-order equation as follows

$$
\begin{aligned}
0 & =\sum_{i=1}^{3} \frac{e_{i} f_{i} \gamma_{i}}{\left(1+\lambda \gamma_{i}\right)^{2}}+\frac{\lambda^{2}}{4} \sum_{i=1}^{3} \frac{h_{i} g_{i} \gamma_{i}}{\left(1+\lambda \gamma_{i}\right)^{2}}-\frac{\lambda}{2} \sum_{i=1}^{3} \frac{e_{i} g_{i} \gamma_{i}}{\left(1+\lambda \gamma_{i}\right)^{2}}-\frac{\lambda}{2} \sum_{i=1}^{3} \frac{h_{i} f_{i} \gamma_{i}}{\left(1+\lambda \gamma_{i}\right)^{2}} \\
& +\sum_{i=1}^{3} \frac{h_{j} f_{i} \gamma_{i}}{\left(1+\lambda \gamma_{i}\right)}-\frac{\lambda}{2} \sum_{i=1}^{3} \frac{h_{i} g_{i} \gamma_{i}}{\left(1+\lambda \gamma_{i}\right)}+h_{4} f_{4}-\frac{\lambda}{2} h_{4} g_{4} .
\end{aligned}
$$

After obtaining the seven roots of (69), we discard the complex roots, and plug the real roots into (14). Finally, we choose the estimate $\hat{\mathbf{y}}$, which fulfills (10). Note that (14) is a CLS estimate of $\mathbf{y}$ with $\mathbf{W}=\mathbf{I}$. Since the optimal $\mathbf{W}^{*}$ depends on the unknown $\mathbf{d}$, the CWLS problem can be solved in a similar way by iteratively updating the weights and the estimates, thus we do not repeat it here.

Appendix 2 Proof of $\mathbf{P}_{\boldsymbol{i}}\left(\left(\mathbf{T}_{\boldsymbol{i}} \mathbf{u}\right) \odot\left(\mathbf{T}_{\boldsymbol{i}} \mathbf{u}\right)\right)=\mathbf{P}_{\boldsymbol{i}} \mathbf{T}_{\boldsymbol{i}}(\mathbf{u} \odot \mathbf{u})$ Recalling that $\mathbf{T}_{i} \mathbf{u}=\mathbf{T}_{i 1} \mathbf{u}-u_{i} \mathbf{1}_{m-\mathrm{i}}, \mathbf{T}_{i} \mathbf{1}_{m}=\mathbf{0}_{M-1}$, and $\mathbf{P}_{i}$
$\mathbf{T}_{i} \mathbf{u}=\mathbf{0}_{M-1}$, we prove that $\mathbf{P}_{i}\left(\left(\mathbf{T}_{i} \mathbf{u}\right) \odot\left(\mathbf{T}_{i} \mathbf{u}\right)\right)$ in (33) is equivalent to $\mathbf{P}_{i} \mathbf{T}_{i}(\mathbf{u} \odot \mathbf{u})$ as follows

$$
\begin{aligned}
\mathbf{P}_{i}\left(\left(\mathbf{T}_{i} \mathbf{u}\right) \odot\left(\mathbf{T}_{i} \mathbf{u}\right)\right) & =\mathbf{P}_{i}\left(\left(\mathbf{T}_{i 1} \mathbf{u}-u_{i} \mathbf{1}_{M-1}\right) \odot\left(\mathbf{T}_{i 1} \mathbf{u}-u_{i} \mathbf{1}_{M-1}\right)\right) \\
& =\mathbf{P}_{i}\left(\left(\mathbf{T}_{i 1} \mathbf{u}\right) \odot\left(\mathbf{T}_{i 1} \mathbf{u}\right)-2 u_{i} \mathbf{T}_{i 1} \mathbf{u}+u_{i}^{2} \mathbf{1}_{M-1}\right) \\
& =\mathbf{P}_{i}\left(\left(\mathbf{T}_{i 1} \mathbf{u}\right) \odot\left(\mathbf{T}_{i 1} \mathbf{u}\right)-2 u_{i}\left(\mathbf{T}_{i 1} \mathbf{u}-u_{i} \mathbf{1}_{M-1}\right)-u_{i}^{2} \mathbf{1}_{M-1}\right) \\
& =\mathbf{P}_{i}\left(\left(\mathbf{T}_{i 1} \mathbf{u}\right) \odot\left(\mathbf{T}_{i 1} \mathbf{u}\right)-2 u_{i} \mathbf{T}_{i} \mathbf{u}-u_{i}^{2} \mathbf{1}_{M-1}\right) \\
& =\mathbf{P}_{i}\left(\left(\mathbf{T}_{i 1} \mathbf{u}\right) \odot\left(\mathbf{T}_{i 1} \mathbf{u}\right)-u_{i}^{2} \mathbf{1}_{M-1}\right) \\
& =\mathbf{P}_{i} \mathbf{T}_{i}(\mathbf{u} \odot \mathbf{u})
\end{aligned}
$$

\section{Appendix 3 Derivation of (35)}

The SVD of $\mathbf{P}_{i} \mathbf{T}_{i}$ is given by $\mathbf{P}_{i} \mathbf{T}_{i}=\mathbf{U}_{i} \boldsymbol{\Lambda}_{i} \mathbf{V}_{i}^{T}$, where $\mathbf{U}_{i}$ is of size $(M-1) \times(M-2)$ and $\mathbf{V}_{i}$ is of size $M \times(M-$ 2 ), which collect the left and right singular vectors corresponding to the $M-2$ nonzero singular values, and $\boldsymbol{\Lambda}_{i}$ is a diagonal matrix with the $M-2$ nonzero singular values on its diagonal. Note that $\mathbf{U}_{i}^{T} \mathbf{U}_{i}=\mathbf{I}_{M-2}, \mathbf{V}_{i}^{T} \mathbf{V}_{i}=\mathbf{I}_{M-2}, \mathbf{V}_{i}^{T} \mathbf{1}_{M}=\mathbf{0}_{M-2} \quad$ and $\mathbf{V}_{i}^{T} \mathbf{u}=\mathbf{0}_{M-2}$. As a result, the nullspace $\mathcal{N}\left(\mathbf{V}_{i}^{T}\right)=\operatorname{span}\left(\mathbf{1}_{M}, \mathbf{u}\right)$, and $\mathcal{R}\left(\mathbf{V}_{i}\right)=\mathcal{R}(\mathbf{U})$. Using the 
SVD and the property of the pseudo-inverse, we can write $\left(\mathbf{P}_{i} \mathbf{T}_{i} \boldsymbol{\Sigma} \boldsymbol{T}_{i}^{T} \mathbf{P}_{i}\right)^{\dagger}$ as

$$
\begin{aligned}
\left(\mathbf{P}_{i} \mathbf{T}_{i} \boldsymbol{\Sigma} \mathbf{T}_{i}^{T} \mathbf{P}_{i}\right)^{\dagger} & =\left(\mathbf{U}_{i} \boldsymbol{\Lambda}_{i} \mathbf{V}_{i}^{T} \boldsymbol{\Sigma} \mathbf{V}_{i} \boldsymbol{\Lambda}_{i} \mathbf{U}_{i}^{T}\right)^{\dagger} \\
& =\left(\boldsymbol{\Lambda}_{i} \mathbf{U}_{i}^{T}\right)^{\dagger}\left(\mathbf{V}_{i}^{T} \boldsymbol{\Sigma} \mathbf{V}_{i}\right)^{-1}\left(\mathbf{U}_{i} \boldsymbol{\Lambda}_{i}\right)^{\dagger} \\
& =\mathbf{U}_{i} \boldsymbol{\Lambda}_{i}^{-1}\left(\mathbf{V}_{i}^{T} \boldsymbol{\Sigma} \mathbf{V}_{i}\right)^{-1} \boldsymbol{\Lambda}_{i}^{-1} \mathbf{U}_{i}^{T} .
\end{aligned}
$$

Plugging (71) and the SVD of $\mathbf{P}_{i} \mathbf{T}_{i}$ into (34), and making use of the property of the pseudo-inverse again, we arrive at

$$
\begin{aligned}
\mathbf{T}_{i}^{T} \mathbf{P}_{i}\left(\mathbf{P}_{i} \mathbf{T}_{i} \boldsymbol{\Sigma} \mathbf{T}_{i}^{T} \mathbf{P}_{i}\right)^{\dagger} \mathbf{P}_{i} \mathbf{T}_{i} & =\mathbf{V}_{i} \boldsymbol{\Lambda}_{i} \mathbf{U}_{i}^{T} \mathbf{U}_{i} \boldsymbol{\Lambda}_{i}^{-1}\left(\mathbf{V}_{i}^{T} \boldsymbol{\Sigma} \mathbf{V}_{i}\right)^{-1} \boldsymbol{\Lambda}_{i}^{-1} \mathbf{U}_{i}^{T} \mathbf{U}_{i} \boldsymbol{\Lambda}_{i} \mathbf{V}_{i}^{T} \\
& =\mathbf{V}_{i}\left(\mathbf{V}_{i}^{T} \boldsymbol{\Sigma} \mathbf{V}_{i}\right)^{-1} \mathbf{V}_{i}^{T} \\
& =\left(\mathbf{V}_{i} \mathbf{V}_{i}^{T} \boldsymbol{\Sigma} \mathbf{V}_{i} \mathbf{V}_{i}^{T}\right)^{\dagger},
\end{aligned}
$$

where $\mathbf{V}_{i} \mathbf{V}_{i}^{T}$ is the projection onto $\mathcal{R}(\mathbf{U})$.

\section{Appendix 4 CRB derivation for localization based on TOA measurements}

We analyze the CRB for jointly estimating $\mathbf{x}$ and $b$ based on (1), and assume $n_{i}$ is Gaussian distributed. The FIM $\mathbf{I}_{1}$ $(\boldsymbol{\theta})$ is employed, where $\theta=\left[\mathbf{x}^{T}, b\right]^{T}$, with entries defined as:

$$
\begin{aligned}
\mathbf{I}_{1}(\boldsymbol{\theta}) & =-E\left[\frac{\partial^{2} \ln p(\mathbf{u} ; \boldsymbol{\theta})}{\partial \boldsymbol{\theta} \partial \boldsymbol{\theta}^{T}}\right] \\
& =\left[\frac{\partial \nu}{\partial \boldsymbol{\theta}}\right]^{T} \mathbf{C}^{-1}\left[\frac{\partial \nu}{\partial \boldsymbol{\theta}}\right],
\end{aligned}
$$

where

$$
\begin{aligned}
& v=\mathbf{d}+b \mathbf{1}_{M}, \\
& \mathbf{C}=\operatorname{diag}\left(\left[\sigma_{1}^{2}, \sigma_{2}^{2}, \ldots, \sigma_{M}^{2}\right]^{T}\right), \\
& =\frac{3 c^{2}}{N \pi^{2} \kappa^{2} \mathrm{SNR}} \operatorname{diag}\left(\left[d_{1}^{2}, d_{2}^{2}, \ldots, d_{M}^{2}\right]^{T}\right), \\
& \frac{\partial v}{\partial b}=\mathbf{1}_{M} \\
& {\left[\frac{\partial v}{\partial x_{l}}\right]_{j}=\frac{x_{l}-x_{l, j}}{\left\|\mathbf{x}-\mathbf{x}_{j}\right\|} .}
\end{aligned}
$$

\section{Appendix 5 CRB derivation for localization based on TDOA measurements}

We analyze the CRB for estimating $\mathbf{x}$ based on (40), and assume $n_{i, j}$ is Gaussian distributed. The FIM $\mathbf{I}_{2}(\mathbf{x})$ for the nonredundant set of TDOA measurements is employed, with entries defined as:

$$
\begin{aligned}
\mathbf{I}_{2}(\mathbf{x}) & =-E\left[\frac{\partial^{2} \ln p\left(\mathbf{r}_{i} ; \mathbf{x}\right)}{\partial \mathbf{x} \partial \mathbf{x}^{T}}\right] \\
& =\left[\frac{\partial \boldsymbol{\mu}_{i}}{\partial \mathbf{x}}\right]^{T} \mathbf{C}_{i}^{-1}\left[\frac{\partial \mu_{i}}{\partial \mathbf{x}}\right],
\end{aligned}
$$

Where

$$
\begin{aligned}
& \mu_{i}=\mathbf{T}_{i 1} \mathbf{d}-d_{i} \mathbf{1}_{M-1}, \\
& {\left[\mathbf{C}_{i}\right]_{k, l}= \begin{cases}\frac{3 c^{2}}{N \pi^{2} \kappa^{2}}\left(\frac{d_{i}^{2}}{\mathrm{SNR}}+\frac{d_{k}^{2}}{\mathrm{SNR}}+\frac{d_{i}^{2} d_{k}^{2}}{\mathrm{SNR}^{2}}\right) & k=l \text { and } k<i \\
\frac{3 c^{2}}{N \pi^{2} \kappa^{2}}\left(\frac{d_{i}^{2}}{\mathrm{SNR}}+\frac{d_{k+1}^{2}}{\mathrm{SNR}}+\frac{d_{i}^{2} d_{k+1}^{2}}{\mathrm{SNR}^{2}}\right) & k=l \text { and } k \geq i, \\
\frac{3 c^{2}}{N \pi^{2} \kappa^{2}} \frac{d_{i}^{2}}{\operatorname{SNR}} & \text { else }\end{cases} }
\end{aligned}
$$

$$
\left[\frac{\partial \mu_{i}}{\partial x_{j}}\right]_{k}=\left\{\begin{array}{ll}
\frac{x_{j}-x_{j, k}}{\left\|\mathbf{x}-\mathbf{x}_{k}\right\|}-\frac{x_{j}-x_{j, i}}{\left\|\mathbf{x}-\mathbf{x}_{i}\right\|^{\prime}}, & k<i \\
\frac{x_{j}-x_{j, k+1}}{\left\|\mathbf{x}-\mathbf{x}_{k+1}\right\|}-\frac{x_{j}-x_{j, i}}{\left\|\mathbf{x}-\mathbf{x}_{i}\right\|}, & k \geq i
\end{array} .\right.
$$

Furthermore, let us define $\mu=\left[\mu_{1}^{T},\left[\mu_{2}^{T}\right]_{2: M}, \ldots,\left[\mu_{M-1}^{T}\right]_{M-1}\right]^{T}$, where $\boldsymbol{\mu}_{i}=\left[\boldsymbol{\mu}_{i, 1}, \ldots\right.$, $\left.\boldsymbol{\mu}_{i, i-1}, \boldsymbol{\mu}_{i, i+1}, \ldots, \boldsymbol{\mu}_{i, M}\right]^{T}$, and $\mathbf{C}$ as the covariance matrix of this full set of TDOA measurements. Then the FIM $\mathbf{I}_{3}$ (x) for the full set can also be derived based on (78) by replacing $\boldsymbol{\mu}_{i}$ and $\boldsymbol{C}_{i}$ with $\boldsymbol{\mu}$ and $\mathbf{C}$, respectively. We can obtain $[\boldsymbol{\mu}]_{k}=\mu_{i, j}$, where $k=(i-1) M-i^{2} / 2-i / 2+j, k \in$ $\{1,2, \ldots, M(M-1) / 2\}, i \in\{1,2, \ldots, M-1\}, j \in\{2,3, \ldots, M\}$ and $j>i$.

Consequently, we achieve

$$
\left[\frac{\partial \boldsymbol{\mu}}{\partial x_{l}}\right]_{k}=\frac{x_{l}-x_{l, j}}{\left\|\mathbf{x}-\mathbf{x}_{j}\right\|}-\frac{x_{l}-x_{l, i}}{\left\|\mathbf{x}-\mathbf{x}_{i}\right\|} \text {. }
$$

In the same way, $[\mathbf{C}]_{k, l}=\operatorname{cov}\left(n_{i, j}, n_{p, q}\right)$, where $l=(p$ 1) $M-p^{2} / 2-p / 2+q, l \in\{1,2, \ldots, M(M-1) / 2\}, p \in\{1,2, \ldots$, $M-1\}, q \in\{2,3, \ldots, M\}$ and $q>p$.

\section{Endnotes}

${ }^{\text {a }}$ The sensor elements of a passive sensor array are equivalent to the anchor nodes here. ${ }^{\mathrm{b}}$ Given the matrix $\mathbf{C}$ of size $n \times r$ and the matrix $\mathbf{D}$ of size $r \times m$ both of rank $r$, then if $\mathbf{A}=\mathbf{C D}$, it holds that $\mathbf{A}^{\dagger}=\mathbf{D}^{\dagger} \mathbf{C}^{\dagger}$ [35].

\section{Acknowledgements}

This research was supported in part by STW under the Green and Smart Process Technologies Program (Project 7976).

\section{Authors' contributions}

All authors read and approved the final manuscript.

\section{Competing interests}

The authors declare that they have no competing interests.

Received: 13 May 2011 Accepted: 26 January 2012

Published: 26 January 2012

\footnotetext{
References

1. Parkinson, B, Spilker J, Jr. (eds.), Global Positioning System: Theory and Application (American Institute of Astronautics and Aeronautics, Washington, DC, 1996)!
} 
2. D Torrieri, Statistical theory of passive location systems. IEEE Trans Aerosp Electron Syst. 20(2), 183-198 (1984)

3. V Chandrasekhar, WK Seah, YS Choo, HV Ee, Localization in underwater sensor networks: survey and challenges, in Proc ACM WUWNet, New York, USA, pp. 33-40 (2006)

4. Y Huang, J Benesty, G Elko, R Mersereati, Real-time passive source localization: a practical linear-correction least-squares approach. IEEE Trans Acoust Speech Signal Process. 9(8), 943-956 (2001)

5. D Li, YH Hu, Least square solutions of energy based acoustic source localization problems, in Proc ICPPW, Montreal, Canada, August, pp. 443-446 (2004)

6. J Caffery Jr, GL Stuber, Subscriber location in CDMA cellular networks. IEEE Trans Veh Technol. 47(2), 406-416 (1998). doi:10.1109/25.669079

7. A Sayed, A Tarighat, N Khajehnouri, Network-based wireless location: challenges faced in developing techniques for accurate wireless location information. IEEE Signal Process Mag. 22(4), 24-40 (2005)

8. S Gezici, Z Tian, G Giannakis, H Kobayashi, A Molisch, H Poor, Z Sahinoglu, Localization via ultra-wideband radios: a look at positioning aspects for future sensor networks. IEEE Signal Process Mag. 22(4), 70-84 (2005)

9. N Patwari, J Ash, S Kyperountas, I AO Hero, R Moses, N Correal, Locating the nodes: cooperative localization in wireless sensor networks. IEEE Signal Process Mag. 22(4), 54-69 (2005)

10. K Cheung, H So, WK Ma, Y Chan, Least squares algorithms for time-ofarrival-based mobile location. IEEE Trans Signal Process. 52(4), 1121-1130 (2004). doi:10.1109/TSP.2004.823465

11. A Savvides, CC Han, MB Strivastava, Dynamic fine-grained localization in adhoc networks of sensors, in Proc ACM MobiCom, Rome, Italy, pp. 166-179 (July 2001)

12. D Niculescu, $B$ Nath, $A d$ hoc positioning system (APS) using $A O A$, in Proc IEEE INFOCOM, vol. 3. San Francisco, CA, USA, pp. 1734-1743 (April 2003)

13. P Bahl, V Padmanabhan, RADAR: an in-building RF-based user location and tracking system, in Proc IEEE INFOCOM, vol. 2. Tel Aviv, Israel, pp. 775-784 (March 2000)

14. Y Wang, G Leus, X Ma, Tracking a mobile node by asynchronous networks, in Proc IEEE SPAWC, San Francisco, CA, USA, pp. 1-5 (June 2011)

15. J Yan, Algorithms for indoor positioning systems using ultra-wideband signals, (Delft University of Technology, 2010) PhD Thesis

16. Z Li, W Trappe, Y Zhang, B Nath, Robust statistical methods for securing wireless localization in sensor networks, in Proc IPSN, Los Angeles, CA, USA, pp. 91-98 (April 2005)

17. S Venkatesh, R Buehrer, A linear programming approach to NLOS error mitigation in sensor networks, in Proc IPSN, Nashville, TN, USA, pp. 301-308 (April 2006)

18. I Guvenc, S Gezici, F Watanabe, H Inamura, Enhancements to linear least squares localization through reference selection and ML estimation, in Proc IEEE WCNC, Las Vegas, NV, USA, pp. 284-289 (April 2008)

19. J Smith, J Abel, Closed-form least-squares source location estimation from range-difference measurements. IEEE Trans Acoust Speech Signal Process. 35(12), 1661-1669 (1987). doi:10.1109/TASSP.1987.1165089

20. B Friedlander, A passive localization algorithm and its accurancy analysis IEEE J Ocean Eng. 12(1), 234-245 (1987)

21. Y Chan, K Ho, A simple and efficient estimator for hyperbolic location. IEEE Trans Signal Process. 42(8), 1905-1915 (1994). doi:10.1109/78.301830

22. P Stoica, J Li, Lecture notes-source localization from range-difference measurements. IEEE Signal Process Mag. 23(6), 63-66 (2006)

23. S Ganeriwal, R Kumar, MB Srivastava, Timing-sync protocol for sensor networks, in Proc ACM SenSys, Los Angeles, CA, USA, pp. 138-149 (Nov 2003)

24. F Chan, H So, Efficient weighted multidimensional scaling for wireless sensor network localization. IEEE Trans Signal Process. 57(11), 4548-4553 (2009)

25. Y Bresler, A Macovski, Exact maximum likelihood parameter estimation of superimposed exponential signals in noise. IEEE Trans Acoust Speech Signal Process. 34(5), 1081-1089 (1986). doi:10.1109/TASSP.1986.1164949

26. A Beck, P Stoica, J Li, Exact and approximate solutions of source localization problems. IEEE Trans Signal Process. 56(5), 1770-1778 (2008)

27. $S$ Zhu, Z Ding, Joint synchronization and localization using TOAs: a linearization based WLS solution. IEEE J Sel Areas Commun. 28(7), 1017-1025 (2010)

28. S Bancroft, An algebraic solution of the GPS equations. IEEE Trans Aerosp Electron Syst. 21(1), 56-59 (1985)
29. H Schau, A Robinson, Passive source localization employing intersecting spherical surfaces from time-of-arrival differences. IEEE Trans Acoust Speech Signal Process. 35(8), 1223-1225 (1987)

30. DH Shin, TK Sung, Comparisons of error characteristics between TOA and TDOA positioning. IEEE Trans Aerosp Electron Syst. 38(1), 307-311 (2002). doi:10.1109/7.993253

31. HC So, YT Chan, F Chan, Closed-form formulae for time-difference-of-arrival estimation. IEEE Trans Signal Process. 56(6), 2614-2620 (2008)

32. M Gillette, $\mathrm{H}$ Silverman, A linear closed-form algorithm for source localization from time-differences of arrival. IEEE Signal Process Lett. 15, 1-4 (2008)

33. A Amar, $\mathrm{G}$ Leus, A reference-free time difference of arrival source localization using a passive sensor array. IEEE SAM 157-160 (October 2010)

34. G Golub, C van Loan, Matrix Computation, (The Johns Hopkins University Press, Baltimore, 1996)

35. S Barnet, Matrices, Methods and Applications, Oxford Applied Mathematics and Computing Science Series, (Clarendon Press, Oxford, 1990)

36. J Smith, J Abel, The spherical interpolation method of source localization. IEEE J Ocean Eng. 12(1), 246-252 (1987). doi:10.1109/JOE.1987.1145217

\section{doi:10.1186/1687-6180-2012-19}

Cite this article as: Wang and Leus: Reference-free time-based localization for an asynchronous target. EURASIP Journal on Advances in Signal Processing 2012 2012:19.

\section{Submit your manuscript to a SpringerOpen ${ }^{\circ}$ journal and benefit from:}

- Convenient online submission

- Rigorous peer review

- Immediate publication on acceptance

- Open access: articles freely available online

- High visibility within the field

- Retaining the copyright to your article

Submit your next manuscript at $\gg$ springeropen.com 